\title{
Adiabatic Theorems for Generators of Contracting Evolutions
}

\section{Journal Article}

\section{Author(s):}

Avron, J.E.; Fraas, M.; Graf, G.M.; Grech, P.

Publication date:

2012-08

Permanent link:

https://doi.org/10.3929/ethz-b-000053820

\section{Rights / license:}

In Copyright - Non-Commercial Use Permitted

\section{Originally published in:}

Communications in Mathematical Physics 314(1), https://doi.org/10.1007/s00220-012-1504-1 


\title{
Adiabatic Theorems for Generators of Contracting Evolutions
}

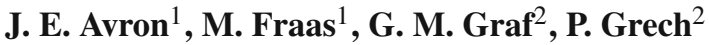 \\ 1 Department of Physics, Technion, 32000 Haifa, Israel \\ 2 Theoretische Physik, ETH Zurich, 8093 Zurich, Switzerland. \\ E-mail: pgrech@itp.phys.ethz.ch
}

Received: 1 July 2011 / Accepted: 17 January 2012

Published online: 30 May 2012 - C Springer-Verlag 2012

\begin{abstract}
We develop an adiabatic theory for generators of contracting evolution on Banach spaces. This provides a uniform framework for a host of adiabatic theorems ranging from unitary quantum evolutions through quantum evolutions of open systems generated by Lindbladians all the way to classically driven stochastic systems. In all these cases the adiabatic evolution approximates, to lowest order, the natural notion of parallel transport in the manifold of instantaneous stationary states. The dynamics in the manifold of instantaneous stationary states and transversal to it have distinct characteristics: The former is irreversible and the latter is transient in a sense that we explain. Both the gapped and gapless cases are considered. Some applications are discussed.
\end{abstract}

\section{Introduction}

We develop a framework for the adiabatic theory of systems whose evolution is governed by a slowly evolving family of linear operators generating a contraction in a Banach space $[1,18,28]$. More precisely, we study equations of the form

$$
\varepsilon \dot{x}(s)=L(s) x(s),
$$

where $L(s)$ is, for any fixed $s$, the generator of a contraction semigroup.

The framework encompasses a wide range of applications from driven stochastic systems generated in a Markovian process, through isolated quantum systems undergoing unitary evolution generated by Hamiltonians, culminating in open quantum systems whose evolution is generated by Lindblad operators.

Adiabatic evolutions have a geometric character. As we shall see, the manifold of instantaneous stationary vectors, namely $\operatorname{ker} L(s)$, has a distinguished complement, with the property that a vector near the former evolves with a velocity in the latter, to leading order in $\varepsilon$. Hence, to lowest order in the adiabatic limit, the vector is parallel transported with the manifold. 


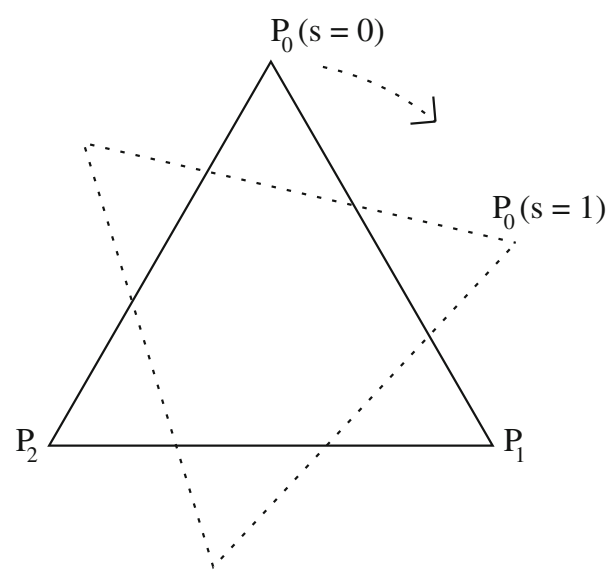

Fig. 1. An example where the set of instantaneous stationary states forms a simplex, here a triangle. The extreme points represent the spectral projections $P_{i}(s), i=0,1,2$. Parallel transport rotates the triangle at time $s=0$ (triangle whose boundary is the full line) to the triangle at time $s=1$ (triangle whose boundary is the dashed line) as a rigid body

Parallel transport may be described more concretely within a particular context, as we will show in the next section. For instance, when vectors represent quantum states, the instantaneous stationary states are transported like points of a rigid body (see Fig. 1).

We consider both the case where $\operatorname{ker} L(s)$ is protected by a gap condition, i.e. 0 is an isolated eigenvalue of $L(s)$, and where it is not.

In the gapped case we give an adiabatic expansion which reveals that the dynamics has distinct characters within the evolving subspace of instantaneous stationary states and transversal to it. Notably, as we shall see, the motion within $\operatorname{ker} L(s)$ is persistent and partly even irreversible, whereas the motion transversal to it is transient in the following sense: Consider the adiabatic evolution over a finite interval, traversed at a slow rate $\varepsilon$; assume that the generator is constant near its endpoints and smooth otherwise, and let the initial state be stationary. Then the distance of the final state from the manifold of stationary states is exceedingly small in $\varepsilon$ (in fact of infinite order: $O\left(\varepsilon^{N}\right)$ for all $N$ ), whereas the distance covered within the manifold is typically $O(1)$ and consists in turn of two parts: A geometric and potentially reversible part, due to parallel transport, and a subleading irreversible correction as large as $O(\varepsilon)$ (see Fig. 2). As we will see by the end of the section, this single result entails contrasting physical consequences for isolated and open quantum systems.

In the gapless case we no longer obtain an expansion, however we prove that the dynamics of the system is constrained to the manifold of instantaneous stationary states and is parallel transported along with the manifold as $\varepsilon \rightarrow 0$. As an application, it generalizes the adiabatic theorem without a gap condition for the Hamiltonian case $([2,10,35])$ to a class of open quantum systems.

Although the framework and the theorems are general and independent of the context, the geometric interpretation and the implications of the theorems may depend on it. It is instructive to illustrate this point for quantum adiabatic theorems. The most familiar version is formulated for the Schrödinger equation, where the state of the system is described by a vector in Hilbert space, and $x$ of Eq. (1) is $|\psi\rangle$. An alternate description could have been given for the von Neumann equation, where the state of the system 


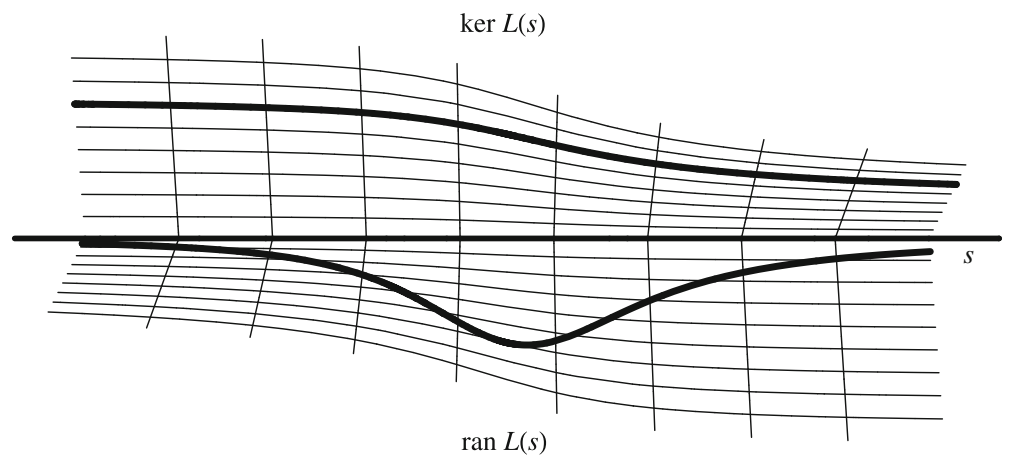

Fig. 2. The straight lines of the upper and lower bundles represent the kernel and the range of $L(s)$, as they change with $s$ and attain (left and right) asymptotes when $L(s)$ does. The thin curves are the result of parallel transport. The thick curves illustrate the motion within the two subspaces, as described in Theorem 6 and Corollary 7. It shows the transient nature of the motion in the range: That part is smaller than any power of $\varepsilon$, when $L(s)$ does not vary

is described by a density matrix, and $x$ is $\rho$, a positive matrix with unit trace. For an isolated system, undergoing unitary evolution, the two descriptions are, in principle, equivalent up to the loss of an overall phase information in $\rho$; nevertheless the elementary formulation and direct proofs of the "standard" adiabatic theorem tend to refer to the Schrödinger context. A formulation in terms of the density matrix is of course a prerequisite towards the formulation of adiabatic theorems for open quantum systems, where only the von Neumann context survives. The unified approach presented here gives such a formulation. When applied to the Schrödinger equation, it has a precursor in [8]; when applied to the von Neumann equation, in [27].

It pays to examine a parallel formulation of the adiabatic theorem for state vectors and density matrices in a simple setting. Consider an isolated, finite dimensional quantum system whose evolution is generated by a slowly varying self-adjoint and non-degenerate Hamiltonian $H(s)$. In the context of pure states, where $x=|\psi\rangle$, the manifold of instantaneous stationary states are the eigenvectors associated to a distinguished eigenvalue $e(s)$ and lie in the kernel of $L(s)=\mathrm{i}(H(s)-e(s))$. Note that $L(s)$ "knows about" the (instantaneous) eigenvalue $e(s)$. The adiabatic theorem then says that the evolution within the spectral subspace is persistent and depending on history; to lowest order, it is geometric and encapsulated in Berry's phase [7]. The evolution transversal to this manifold is transient and non-geometric and describes tunneling to eigenvectors of different eigenvalues.

In the context of density matrices, $x=\rho$, the generator of adiabatic evolution $L(s)$ is the adjoint action of $H(s)$, namely $L(s) \rho(s)=-\mathrm{i}[H(s), \rho(s)]$. This generator, being invariant to the replacement $H(s) \rightarrow H(s)-e(s)$, has no information on the distinguished spectral subspace of $H(s)$. Consequently, the manifold of instantaneous stationary states (in the simplest setting we consider) is a simplex whose extreme points are the (instantaneous) spectral projections, see Fig. 1. In this picture, Berry's phase gets lost; however, the associated curvature remains hidden in the motion transversal to the manifold, as revealed in some instances of linear response theory, like for the quantum Hall effect (see also [5]).

We finally consider a class of open quantum systems which, though not Hamiltonian, preserve the Hamiltonian. The generator of the dynamics, called a dephasing Lindbladian, retains the above simplex as its manifold of instantaneous stationary states. If the 
initial data start at a vertex, the motion within the manifold of stationary states simply follows the parametrically rotating vertex (see Fig. 1)-this being the geometric part arising at lowest order-; but to next order the motion is irreversible, non-geometric and directed away from the vertex. It is interpreted as tunneling, in the sense of quantum transitions between states protected by an energy gap, which may but need not be, a coherent process.

The anticipated, contrasting consequences are now evident. By the general result stated earlier, and under its conditions, for systems undergoing unitary evolution tunneling is reversible, since it eventually dwindles to a remainder of infinite order, while for systems governed by a dephasing Lindbladian, tunneling is irreversible and comparatively large, $O(\varepsilon)$.

The plan of the paper is as follows. In Sect. 2 we describe the general adiabatic theorems and the properties of parallel transport. In Sect. 3 we apply these results to unitary and Lindbladian quantum systems, as well as to driven stochastic processes. All proofs, except for a few short ones, are assembled in Sect. 4.

\section{General Results}

In the general scheme mentioned in the Introduction the state space is a Banach space and the generators are those of contraction semigroups. We shall present two adiabatic theorems which, like their Hamiltonian counterparts, either rely on a spectral gap [6,19] or forgo it [2]. Both depend on the notion of parallel transport. Some preliminaries, like the existence of the evolution and the definition of parallel transport, shall be dealt with first.

2.1. Preliminaries. Propagator. We consider the evolution (1) with time-dependent generators $L(s)$, possibly unbounded, and state sufficient conditions for the existence of the propagator on a Banach space $\mathcal{B}$.

Definition 1. Operators $L(s),(0 \leq s \leq 1)$ on $\mathcal{B}$ are called a $C^{k}$-family if: $L(s)$ are closed operators with a common dense domain $D$ and the function $L$, taking values in the Banach space of bounded operators $D \rightarrow \mathcal{B}$, is $k$-times differentiable in s. Here $D$ is endowed with the graph norm of $L(s)$ for any fixed $s$.

Lemma 1. Let $L(s),(0 \leq s \leq 1)$ be a $C^{1}$-family and, for each $s$, the generator of a contraction semigroup on $\mathcal{B}$. Then there exist operators $U_{\varepsilon}\left(s, s^{\prime}\right): \mathcal{B} \rightarrow \mathcal{B},\left(0 \leq s^{\prime} \leq\right.$ $s \leq 1)$ with $U_{\varepsilon}\left(s, s^{\prime}\right) D \subset D, U_{\varepsilon}(s, s)=\mathbb{1}$ and

$$
\varepsilon \frac{\partial}{\partial s} U_{\varepsilon}\left(s, s^{\prime}\right) x=L(s) U_{\varepsilon}\left(s, s^{\prime}\right) x, \quad(x \in D) .
$$

For $x \in D$ the unique solution $x(s) \in D$ of (1) with initial data $x\left(s^{\prime}\right)=x$ is $x(s)=$ $U_{\varepsilon}\left(s, s^{\prime}\right) x$. Moreover,

$$
\left\|U_{\varepsilon}\left(s, s^{\prime}\right)\right\| \leq 1
$$

and

$$
\varepsilon \frac{\partial}{\partial s^{\prime}} U_{\varepsilon}\left(s, s^{\prime}\right) x=-U_{\varepsilon}\left(s, s^{\prime}\right) L\left(s^{\prime}\right) x, \quad(x \in D) .
$$

We will call $U_{\varepsilon}\left(s, s^{\prime}\right) x$ a solution of (1) even for $x \notin D$. 
Remark 1. By definition ([32], Sect. X.8), a contraction semigroup is strongly continuous. Its generator is thus closed and densely defined.

Remark 2. Suppose, in alternative to the hypothesis of the lemma, that the generator $L(s)$ is bounded and strongly continuous, and that $\varepsilon=1$. Then the propagator exists and is bounded (but not necessarily by 1), uniformly in $0 \leq s^{\prime}, s \leq 1$ [22].

Parallel transport. In adiabatic evolutions the manifold of instantaneous stationary states associated to $\operatorname{ker} L(s)$ plays a distinguished role. This motivates our interest in families of projections. We consider $P(s): \mathcal{B} \rightarrow \mathcal{B},(0 \leq s \leq 1)$ to be any $C^{1}$-family of projections in the norm sense. Let $\dot{P}(s)=d P(s) / d s$. Then the parallel transport $T\left(s, s^{\prime}\right): \mathcal{B} \rightarrow \mathcal{B}$ is defined by

$$
\begin{aligned}
\frac{\partial}{\partial s} T\left(s, s^{\prime}\right) & =[\dot{P}(s), P(s)] T\left(s, s^{\prime}\right), \\
T\left(s^{\prime}, s^{\prime}\right) & =\mathbb{1} .
\end{aligned}
$$

It satisfies $P(s) T\left(s, s^{\prime}\right)=T\left(s, s^{\prime}\right) P\left(s^{\prime}\right)$ and hence respects the ranges of $P(s)$. Parallel transport is thus a perfect adiabatic evolution: no transitions from the bundle of projections $P(s)$ to that of the complementary projections $Q(s)=\mathbb{1}-P(s)$, nor vice versa.

A characterization of parallel transport, given in terms of sections $x(s) \in \operatorname{ran} P(s)$, states that the projected velocity vanishes:

$$
x(s)=T(s, 0) x(0) \Leftrightarrow P(s) \dot{x}(s)=0,
$$

and likewise for $Q$ in place of $P$. Indeed, for such sections $\dot{x}=\dot{P} x+P \dot{x}$ and Eq. (5) reduces to

$$
\frac{\partial}{\partial s} T\left(s, s^{\prime}\right) x\left(s^{\prime}\right)=\dot{P}(s) T\left(s, s^{\prime}\right) x\left(s^{\prime}\right)
$$

by $P \dot{P} P=0$; hence the contention Eq. (6).

The parallel transport determined by the dual projections $P(s)^{*}: \mathcal{B}^{*} \rightarrow \mathcal{B}^{*}$ is

$$
T^{*}\left(s, s^{\prime}\right)=\left(T\left(s^{\prime}, s\right)\right)^{*},
$$

as can be seen from Eq. (5). Observe that unless $\mathcal{B}$ and $\mathcal{B}^{*}$ coincide, the notion of orthogonal projection does not make sense a priori. In the applications both projections, orthogonal and otherwise, play a role.

It is often the case that open systems evolve towards a unique equilibrium state or a steady state. This situation is associated with rank 1 projections with special properties (see Lemma 4 and Example 3 below) and motivates the interest in this class.

Lemma 2. Let $P(s)$ be a $C^{1}$-family of rank 1 projections. If $\operatorname{ker} P(s)$ is independent of $s$, then $\dot{P}(s)$ vanishes on $\operatorname{ker} P(s)$ and $P(s)=T\left(s, s^{\prime}\right) P\left(s^{\prime}\right)$.

Note that, without making additional assumptions (e.g. that $P(s)$ is an orthogonal projection), parallel transport is not guaranteed to be a contraction. By Remark 2, one can only conclude that

$$
\sup _{0 \leq s^{\prime}, s \leq 1}\left\|T\left(s, s^{\prime}\right)\right\|<\infty .
$$


2.2. States. States of a physical system often enjoy more properties than mere vectors in a Banach space. The additional structure we will introduce allows for further geometric properties of parallel transport. To mark the difference with the previous and the following subsection, we shall denote states by $\rho$, rather than by $x$. The fundamental objects, however, are the observables, denoted by $a$, and their algebra $\mathcal{A}$.

In the following let $\mathcal{B}=\mathcal{A}^{*}$ be the dual of a $C^{*}$-algebra with identity $\mathcal{A}$. We consider a second $C^{*}$-algebra $\widetilde{\mathcal{A}}$ and bounded linear maps $\Phi: \mathcal{A} \rightarrow \widetilde{\mathcal{A}}$ enjoying

(i) $\Phi$ is positive $(\Phi \geq 0): a \geq 0 \Rightarrow \Phi a \geq 0$;

(ii) $\Phi$ is normalized: $\Phi(\mathbb{1})=\overline{\mathbb{1}}$.

The maps satisfy $\|\Phi\|=1$ ([11], Cor. 3.2.6) and form a norm closed convex set. For $\widetilde{\mathcal{A}}=\mathbb{C}$ one is considering linear functionals, denoted $\rho \in \mathcal{A}^{*}$, and (i, ii) define states, $\rho \in \mathcal{A}_{+, 1}^{*}$. (The subscripts indicate that the functionals are positive and normalized.) For $\widetilde{\mathcal{A}}=\mathcal{A}$, the dual maps $\Phi^{*}: \mathcal{A}^{*} \rightarrow \mathcal{A}^{*}$ satisfy the corresponding properties (i) $\rho \geq 0 \Rightarrow \Phi^{*} \rho \geq 0$ and (ii) $\left(\Phi^{*} \rho\right)(\mathbb{1})=\rho(\mathbb{1})$. We call them state preserving maps. By duality,

$$
\left\|\Phi^{*}\right\|=1
$$

The maps $\Phi$ and $\Phi^{*}$ then refer to the Heisenberg and the Schrödinger picture, (with $\Phi$ acting on observables and $\Phi^{*}$ acting on states). We will consider state preserving maps which are projections $\mathcal{P}: \mathcal{A}^{*} \rightarrow \mathcal{A}^{*}$. (For economy of notation we omit the star and write $\mathcal{P}_{*}$ for the predual, if need arises.) Associated to them are the states in their ranges, $\mathcal{S}:=\mathcal{A}_{+, 1}^{*} \cap \operatorname{ran} \mathcal{P}$. Such projections naturally arise through the mean ergodic theorem ([16], Thm. 18.6.1) as projections on stationary states of state preserving semigroups $\Phi_{t}^{*}$,

$$
\mathcal{P}=\lim _{\gamma \downarrow 0} \gamma \int_{0}^{\infty} \mathrm{e}^{-\gamma t} \Phi_{t}^{*} d t,
$$

provided the limit exists in norm.

Remark 3. In case $\mathcal{A}$ does not have an identity, we obtain $\widehat{\mathcal{A}}$ by adjoining one ([11], Def. 2.1.6). We consider maps defined on $\widehat{\mathcal{A}}$ satisfying (i, ii), provided they are compatible with the adjunction. More precisely, we consider linear functionals $\rho \in \widehat{\mathcal{A}}^{*}$ (and in particular, states), provided they arise by canonical extension from $\rho \in \mathcal{A}$ ([11], p. 52). Of a state preserving map it is then required to be so also w.r.t. the amended sense of states.

Example 1. The compact operators $\mathcal{A}=\operatorname{Com}(\mathcal{H})$ on a Hilbert space $\mathcal{H}$ form a $\mathcal{C}^{*}$-algebra (an identity may be adjoined). Its dual $\mathcal{A}^{*}=\mathcal{J}_{1}(\mathcal{H})$ are the trace class operators. Any $\rho \in \mathcal{J}_{1}(\mathcal{H})$ is a state if $\rho \geq 0$ and $\operatorname{tr} \rho=1$. An example of a state preserving projection $\mathcal{P}$ is

$$
\mathcal{P} \rho=\sum_{i} P_{i} \rho P_{i}
$$

where the $P_{i}$ are an orthogonal partition of unity on $\mathcal{H}$. As required by the definition of state preserving maps, $\mathcal{P}$ is the dual of a positive normalized map $\mathcal{P}_{*}$ on $\operatorname{Com}(\mathcal{H})$. In fact, $\mathcal{P}_{*}$ also acts by (11). 
The following proposition is concerned with families of projections $\mathcal{P}(s)$ and, more precisely, with the corresponding parallel transport $\mathcal{T}\left(s, s^{\prime}\right)$, determined by Eq. (5), and states $\mathcal{S}(s)$ : The action of the former on the latter is that of a rigid motion. In the context of Example 1 the proposition is illustrated in Fig. 1.

Proposition 3 (Rigid transport). Let the $C^{1}$-family of projections $\mathcal{P}(s): \mathcal{A}^{*} \rightarrow \mathcal{A}^{*}$, $(0 \leq s \leq 1)$ be state preserving. Then $\mathcal{T}\left(s, s^{\prime}\right) \mathcal{P}\left(s^{\prime}\right)$ is also state preserving. In particular, $\mathcal{T}\left(s, s^{\prime}\right)$ maps

- $\mathcal{S}\left(s^{\prime}\right)$ to $\mathcal{S}(s)$ isometrically;

- (isolated) extreme points of $\mathcal{S}\left(s^{\prime}\right)$ to corresponding ones of $\mathcal{S}(s)$.

Moreover, if $\rho(s) \in \operatorname{ran} \mathcal{P}(s)$, depending continuously on $s$, is an isolated extreme point of $\mathcal{S}(s)$, then $\rho(s)=\mathcal{T}\left(s, s^{\prime}\right) \rho\left(s^{\prime}\right)$.

Example 2 (continuing Example 1). With respect to the Hilbert-Schmidt inner product induced by the inclusion $\mathcal{J}_{1}(\mathcal{H}) \subset \mathcal{J}_{2}(\mathcal{H})$, the projection $\mathcal{P}$ is orthogonal and the transport $\mathcal{T}$ unitary. These two properties are seen from the following consideration: Since $\mathcal{J}_{1}(\mathcal{H}) \subset \operatorname{Com}(\mathcal{H})$, the action of $\mathcal{P}_{*}: \operatorname{Com}(\mathcal{H}) \rightarrow \operatorname{Com}(\mathcal{H})$ can be compared with that of $\mathcal{P}: \mathcal{P}_{*}$ preserves $\mathcal{J}_{1}(\mathcal{H})$ and $\mathcal{P}_{*} \uparrow \mathcal{J}_{1}(\mathcal{H})=\mathcal{P}$. We thus have $\mathcal{T}_{*}\left(s, s^{\prime}\right)\left\lceil\mathcal{J}_{1}(\mathcal{H})=\mathcal{T}\left(s, s^{\prime}\right)\right.$ by $(5)$, besides $\left(\mathcal{T}\left(s, s^{\prime}\right) \rho\right)\left(\mathcal{T}_{*}\left(s, s^{\prime}\right) a\right)=\rho(a)$ by $(8)$.

The example is illustrated in Fig. 1. The motion is rigid in the metrics of both $\mathcal{J}_{1}(\mathcal{H})$ and $\mathcal{J}_{2}(\mathcal{H})$. Explicitly, if $\rho(s)=\sum \lambda_{j} P_{j}(s)$ then $\rho\left(s^{\prime}\right)=\sum \lambda_{j} P_{j}\left(s^{\prime}\right)$ for the same $\lambda_{j}$, while the projections retain their distances in both norms.

We conclude with a consideration about rank 1 projections, which is linked to Lemma 2. In the present setting its hypothesis is satisfied:

Lemma 4. Consider state preserving projections $\mathcal{P}$ of rank 1 . Then $\operatorname{ran} \mathcal{P}_{*}=\operatorname{span}\{\mathbb{1}\}$ and $\operatorname{ker} \mathcal{P}$ is independent of $\mathcal{P}$. In particular, if $\mathcal{P}(s)$ is a $C^{1}$-family of such projections, then $\mathcal{P}(s)=\mathcal{T}\left(s, s^{\prime}\right) \mathcal{P}\left(s^{\prime}\right)$ and $\rho(s)=\mathcal{T}\left(s, s^{\prime}\right) \rho\left(s^{\prime}\right)$, where $\rho(s)$ is the unique state in $\operatorname{ran} \mathcal{P}(s)$.

Example 3. Let $\mathcal{A}=\operatorname{Com}(\mathcal{H})$ and let $\rho_{0} \in \mathcal{J}_{1}(\mathcal{H})$ be a state. Then the rank 1 projection $\mathcal{P}: \rho \mapsto(\operatorname{tr} \rho) \rho_{0}$ is state preserving with $\operatorname{ker} \mathcal{P}=\{\rho \mid \operatorname{tr} \rho=0\}$, and $\mathcal{P}_{*}: a \mapsto$ $\operatorname{tr}\left(\rho_{0} a\right) \mathbb{1}$. If $\rho_{0}=\rho_{0}(s)$ is a $C^{1}$-family, then $\dot{\mathcal{P}}(s) \rho=(\operatorname{tr} \rho) \dot{\rho}_{0}(s)$ and the statements of the lemma are evident. Note however that, in contrast to the projection (11), the actions of $\mathcal{P}$ and $\mathcal{P}_{*}$ are different. Hence $\mathcal{P}$ is not orthogonal in $\mathcal{J}_{2}(\mathcal{H})$.

2.3. An adiabatic theorem in presence of a gap. We assume that 0 is an isolated point of the spectrum of $L$, which is what we mean by a gap. Then, for small $\varepsilon$, the differential equation forces a fast time scale of order $O\left(\varepsilon^{-1}\right)$ on vectors transverse to the null space ker $L(s)$. That scale reflects itself in a fast motion, consisting of oscillations and decay. By contrast on vectors in the null space the dynamics is slow by $\dot{x}=0$. Nevertheless these vectors leak out of that subspace, because it is itself changing with $s$. The leakage however remains of order $O(\varepsilon)$, as shown by Theorem 9 below. A complementary result, Theorem 6 , constructs a "slow manifold", where solutions $x(s)$ remain suitably close to $\operatorname{ker} L(s)$ and the time scale is $O(1)$. Before presenting the two results, which are illustrated in Fig. 3, we need to specify the transversal subspace complementing $\operatorname{ker} L(s)$. 


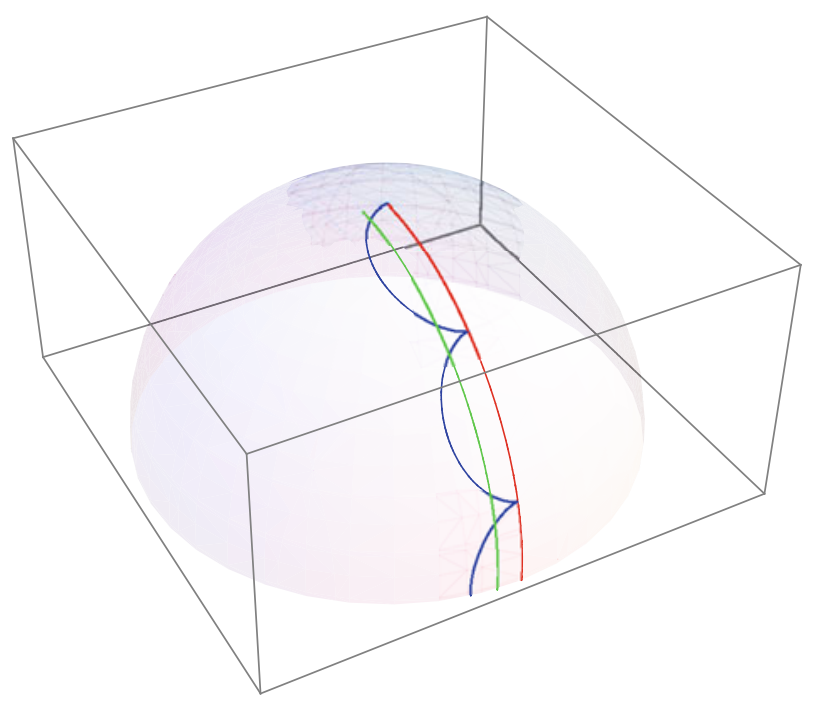

Fig. 3. The figure shows the result of a computation of the unitary adiabatic evolution of a qubit, see Subsect. 3.3 for details. The state is represented as a point on the Bloch sphere, Eq. (35). The (red) meridian shows the manifold of instantaneous stationary states, i.e. ker $L$. The parametrization corresponds to uniform speed along this path. The "slow manifold" is represented by the (green) curve essentially parallel to the (red) meridian. An orbit is shown by the (blue) cycloid. Note that the initial conditions do not lie on the slow manifold $\left(b_{1}(0) \neq 0\right.$ when $\dot{P}(0) \neq 0$ ). This is the reason for the large oscillations (color in electronic version only)

The general assumptions on $L=L(s),(0 \leq s \leq 1)$ are

Hypothesis 1. L is the generator of a contraction semigroup on a Banach space $\mathcal{B}$.

As a consequence one has

Proposition 5. The null space and the range of $L$, the generator of a contraction semigroup, are transversal in the sense that

$$
\operatorname{ker} L \cap \operatorname{ran} L=\{0\} .
$$

The issue whether the two spaces complement each other is covered by the next hypothesis.

Hypothesis 2. The range of $L$ is closed and complementary to the (closed) null space of $L$ :

$$
\mathcal{B}=\operatorname{ker} L \oplus \operatorname{ran} L,
$$

and the corresponding projections are denoted $\mathbb{1}=P+Q$.

Remark 4. We recall that $\mathcal{B}_{1} \oplus \mathcal{B}_{2}$ is the notation for the sum $\mathcal{B}_{1}+\mathcal{B}_{2}$ of subspaces $\mathcal{B}_{i} \subset \mathcal{B}(i=1,2)$ in the case that any vector $x$ in the sum admits a unique decomposition $x=x_{1}+x_{2}$ with $x_{i} \in \mathcal{B}_{i}$. Any two among the statements " $\mathcal{B}_{i}(i=1,2)$ are closed", " $\mathcal{B}_{1}+\mathcal{B}_{2}$ is closed", and " $P_{i}: x \mapsto x_{i}(i=1,2)$ are bounded" imply the third.

Hypothesis 3. $L(s)$ is a $C^{k}$-family for which 0 remains a uniformly isolated eigenvalue. 
Remark 5. We will see by Hypothesis 2 that zero is either in the resolvent set or an isolated point of the spectrum $\sigma(L)$. In the latter case, by Hypothesis 3, the gap is then assumed to be uniform. The restriction $L \uparrow \operatorname{ran} L$ has a bounded inverse, denoted by $L^{-1}$, and $P(s)$ and $L(s)^{-1}$ are $C^{k}$ in norm.

Remark 6. We will give sufficient conditions for Hypothesis 2 in Subsect. 2.5. For short, it is the regular case, given Hypothesis 1 .

For $\varepsilon=0$, Eq. (1) requires $x(s) \in \operatorname{ker} L(s)$. For small $\varepsilon$ the differential equation admits solutions which remain close to $\operatorname{ker} L(s)$. The construction of the "slow manifold" reduces to a differential equation for the slow variables only, with the fast ones providing the inhomogeneity. The latter, rather than being governed by a further, coupled differential equation, are enslaved to the solution at lower orders. More precisely, the solutions are described as follows.

Theorem 6 (Slow manifold expansion). Let $L(s)$ be a $C^{N+2}$-family of operators satisfying Hypotheses 1-3. Then

1. The differential equation $\varepsilon \dot{x}=L(s) x$ admits solutions of the form

$$
x(s)=\sum_{n=0}^{N} \varepsilon^{n}\left(a_{n}(s)+b_{n}(s)\right)+\varepsilon^{N+1} r_{N}(\varepsilon, s)
$$

with

- $a_{n}(s) \in \operatorname{ker} L(s), b_{n}(s) \in \operatorname{ran} L(s)$.

- initial data $x(0)$ specified by arbitrary $a_{n}(0) \in \operatorname{ker} L(0), r_{N}(\varepsilon, 0) \in \mathcal{B}$; however, the $b_{n}(0)$ are determined below by the $a_{n}(0)$ and together define the "slow manifold".

2. The coefficients are determined recursively through $(n=0, \ldots, N)$ :

$$
\begin{aligned}
b_{0}(s) & =0, \\
a_{n}(s) & =T(s, 0) a_{n}(0)+\int_{0}^{s} T\left(s, s^{\prime}\right) \dot{P}\left(s^{\prime}\right) b_{n}\left(s^{\prime}\right) d s^{\prime}, \\
b_{n+1}(s) & =L(s)^{-1} \dot{P}(s) a_{n}(s)+L(s)^{-1} Q(s) \dot{b}_{n}(s) .
\end{aligned}
$$

3. The remainder is

$$
\begin{aligned}
r_{N}(\varepsilon, s)= & U_{\varepsilon}(s, 0) r_{N}(\varepsilon, 0)+b_{N+1}(s)-U_{\varepsilon}(s, 0) b_{N+1}(0) \\
& -\int_{0}^{s} U_{\varepsilon}\left(s, s^{\prime}\right) \dot{b}_{N+1}\left(s^{\prime}\right) d s^{\prime}
\end{aligned}
$$

where $U_{\varepsilon}\left(s, s^{\prime}\right)$ is the propagator described in Lemma 1. It is uniformly bounded in $\varepsilon$, if $r_{N}(\varepsilon, 0)$ is:

$$
\sup _{s}\left\|r_{N}(\varepsilon, s)\right\| \leq C_{N} \sum_{n=0}^{N}\left\|a_{n}(0)\right\|+\left\|r_{N}(\varepsilon, 0)\right\|,
$$

where $C_{N}$ depends on the family. 
Explicitly: for $a_{1}(0)=0$ we have

$$
\begin{aligned}
& a_{0}(s)=T(s, 0) a_{0}(0), \\
& b_{1}(s)=L(s)^{-1} \dot{P}(s) a_{0}(s), \\
& a_{1}(s)=\int_{0}^{s} T\left(s, s^{\prime}\right) \dot{P}\left(s^{\prime}\right) L\left(s^{\prime}\right)^{-1} \dot{P}\left(s^{\prime}\right) a_{0}\left(s^{\prime}\right) d s^{\prime} .
\end{aligned}
$$

Corollary 7. If $L(s)$ is constant on an interval $I \subset[0,1]$, then

$$
b_{n}(s)=0, \quad(s \in I) .
$$

Proof. This follows recursively from (16) by $\dot{P}(s)=0$.

Corollary 8. If $P(s)$ are rank 1 projections and $\operatorname{ker} P(s)$ is independent of $s$, then $a_{n}(s)=T(s, 0) a_{n}(0)$.

This is the case in Example 3. See Subsect. 3.2 for an application.

Proof. In Eq. (15) we have $\dot{P}\left(s^{\prime}\right) b_{n}\left(s^{\prime}\right)=0$ in view of Lemma 2 and of $b_{n}\left(s^{\prime}\right) \in \operatorname{ran} L=$ ker $P$.

In Theorem 6 the initial data $x(0)=P(0) x(0)+Q(0) x(0)$ is such that the first (slow) part is arbitrary, and it prescribes the second (fast) part, up to a remainder. The general case that both parts of the initial condition are arbitrary is addressed by a result on the decoupling of the slow variables from the fast variables:

Theorem 9 (Decoupling). Let $L(s)$ be a $C^{2}$-family satisfying the assumptions of Theorem 6 . Then for any solution $x(s)$ of Eq. (1),

$$
\|P(s) x(s)-T(s, 0) P(0) x(0)\| \leq C \varepsilon\|x(0)\|, \quad(0 \leq s \leq 1),
$$

where $C$ depends on the family.

Remark 7. No statement about the fast part, $Q(s) x(s)$, is made. The theorem may in particular be applied to the difference $\tilde{x}(0)=Q(0) \tilde{x}(0)$ of initial conditions sharing the same slow part; in this case, $\|P(s) \tilde{x}(s)\| \leq C \varepsilon\|\tilde{x}(0)\|$.

The proof of Theorem 9 will depend on the following result. We consider linear forms $\varphi \in \mathcal{B}^{*}$, the dual of $\mathcal{B}$. The duality bracket is $\langle\varphi, x\rangle$.

Proposition 10 (Adiabatic invariants). Let $L(s)$ be a $C^{1}$-family as above. Suppose the family $\varphi(s) \in \mathcal{B}^{*},(0 \leq s \leq 1)$ satisfies

$$
\varphi(s) \in \operatorname{ker} L^{*}(s), \quad \dot{\varphi}(s) \in \operatorname{ran} L^{*}(s) .
$$

Then $\varphi$ is an approximate adiabatic invariant in the sense that for any solution $x(t)$ of Eq. (1),

$$
\left.\langle\varphi, x\rangle\right|_{0} ^{s}=\varepsilon \int_{0}^{s}\left\langle L^{*-1} \dot{\varphi}, \dot{x}\right\rangle d s^{\prime}
$$

Assuming $C^{2}$-regularity, the expression is bounded as

$$
|\langle\varphi, x\rangle|_{0}^{s} \mid \leq C \varepsilon\|\varphi(s)\|\|x(0)\|,
$$

where $C$ depends on the family $L(s)$. 
2.4. An adiabatic theorem in absence of a gap. In the absence of a gap a weaker replacement for the previous theorem is provided by the following result, which relies on Hypothesis 1 and replaces Hypotheses 2-3 by

\section{Hypothesis 2;.}

$$
\mathcal{B}=\operatorname{ker} L \oplus \overline{\operatorname{ran} L} .
$$

Hypothesis 3. $L(s)$ is a $C^{1}$-family.

Theorem 11 (Gapless). Let L(s) satisfy Hypotheses 1, 2', and 3' for all $0 \leq s \leq 1$ and let $P(s)$, for almost all $s$, be the projection associated to $\operatorname{ker} L(s)$ in the decomposition (24); moreover let $P(s)$ be defined for all $0 \leq s \leq 1$ and $C^{1}$ as a bounded operator on $\mathcal{B}$. Then the solution of $\varepsilon \dot{x}=L(s) x$ with initial data $x(0)=P(0) x(0)$ satisfies

$$
\sup _{s \in[0,1]}\|x(s)-T(s, 0) x(0)\| \rightarrow 0, \quad(\varepsilon \rightarrow 0) .
$$

Remark 8. The theorem generalizes the Hamiltonian adiabatic theorem in the absence of gap $[2,10]$ to the non-self-adjoint case. Actually, the $C^{2}$-regularity of $P(s)$ assumed there is relaxed here to $C^{1}$ thanks to a remark by Elgart, reported in [35].

Remark 9. The "almost all" formulation [10,35] allows for eigenvalue crossings.

Proposition 10 has the following variant in the gapless case.

Proposition 12. Let $L(s)$ be a $C^{1}$-family satisfying Hypothesis 1. Suppose the family $\varphi(s) \in \mathcal{B}^{*},(0 \leq s \leq 1)$ satisfies

$$
\varphi(s) \in \operatorname{ker} L^{*}(s), \quad \dot{\varphi}(s)=L^{*}(s) \phi(s)
$$

with uniformly bounded $\phi(s)$ and $\dot{\phi}(s)$. Then

$$
|\langle\varphi, x\rangle|_{0}^{s} \mid \leq 3 \varepsilon \sup _{0 \leq s^{\prime} \leq 1}\left(\left\|\phi\left(s^{\prime}\right)\right\|+\left\|\dot{\phi}\left(s^{\prime}\right)\right\|\right)\|x(0)\| .
$$

2.5. Complementarity of subspaces. In this subsection we will give sufficient conditions for the complementarity Hypothesis 2 in relation with a spectral gap, and 2' in its absence. As a help to gauge them, note that both are false if 0 is an eigenvalue of $L$ with non-vanishing eigennilpotent, but they hold true for a self-adjoint operator $L$ on a Hilbert space if 0 is an isolated resp. non-isolated point of its spectrum.

The two subspaces in Eqs. $(13,24)$ are transversal,

$$
\operatorname{ker} L \cap \overline{\operatorname{ran} L}=\{0\},
$$

as a consequence of Hypothesis 1, as we shall see. However they may fail to generate $\mathcal{B}$ without further hypotheses. Such hypotheses are given in two lemmas corresponding to the two cases. There, a prime indicates a hypothesis tailored to the second, gapless case; a sufficient condition for an earlier hypothesis is noted by an added roman numeral.

Counterexamples matching the two cases are also given. Related results are found in ([16], Thm. 18.8.3).

Lemma 13. Let $\mathcal{B}$ be a Banach space and $L$ a closed operator on $\mathcal{B}$. Assume, besides Hypothesis 1, that 
(H2i) If 0 is in the spectrum, $\sigma(L)$, then 0 is a discrete eigenvalue.

Then $\mathcal{B}=\operatorname{ker} L \oplus \operatorname{ran} L, c f . E q$. (13).

Property (H2i) implies that L is Fredholm, and hence

(H2ii) L is semi-Fredholm.

In conjunction with Hypothesis 1, Properties (H2i) and (H2ii) are equivalent.

Remark 10. By definition, a discrete eigenvalue is an isolated point $\lambda$ of the spectrum, the Riesz projection of which,

$$
P=-\frac{1}{2 \pi \mathrm{i}} \oint_{\Gamma}(L-z)^{-1} d z,
$$

is finite-dimensional. Here $\lambda$ is the only point of the spectrum encircled by $\Gamma$.

Remark 11. Hypothesis (H2i) is trivially satisfied if $\operatorname{dim} \mathcal{B}<\infty$.

Remark 12. We recall that $L$ is semi-Fredholm iff $\operatorname{ran} L$ is closed and $\operatorname{ker} L$ or $\mathcal{B} / \operatorname{ran} L$ are finite-dimensional. If both are, $L$ is called Fredholm.

Example 4. Assumption (H2i) can not be omitted from Lemma 13 if the splitting (13) is to be ensured. In fact in ([24], Thm. 2.2) an example is given of a non-trivial generator $L$ of contraction semigroup with trivial null space, yet with $\sigma(L)=\{0\}$. Hence $(\mathrm{H} 2 \mathrm{i})$ fails there. By the equivalence with (H2ii), $\operatorname{ran} L$ is not closed, spoiling (13).

Lemma 14. Let $\mathcal{B}$ be a Banach space and $L$ a closed operator on $\mathcal{B}$. Assume, besides Hypothesis 1, that

(H2'i) $\mathcal{B}=\operatorname{ker} L+\overline{\operatorname{ran} L}$.

Then $\mathcal{B}=\operatorname{ker} L \oplus \overline{\operatorname{ran} L}, c f . E q$. (24).

Moreover, if $\operatorname{ker} L+\overline{\operatorname{ran} L}$ is closed and $\mathcal{B}$ reflexive, then (H2'i) follows from Hypothesis 1 .

Recall that, by definition, $\mathcal{B}$ is reflexive if $\mathcal{B}^{* *}=\mathcal{B}$.

Example 5. Consider the operator $L$ defined by $(L f)(x)=-x f(x)$ for $f \in L^{\infty}(0,1)=$ $\mathcal{B}$. Obviously, $L$ has trivial kernel and $\left(\mathrm{e}^{L t} f\right)(x)=\mathrm{e}^{-x t} f(x)$, which makes $L$ the generator of a contraction semigroup. However, for $1 \equiv g \in L^{\infty}(0,1)$ one has

$$
\|g-L f\|_{L^{\infty}} \geq 1, \quad\left(f \in L^{\infty}(0,1)\right) .
$$

Thus $\overline{\operatorname{ran} L}$ is a proper subspace of $L^{\infty}(0,1)$. In relation with Lemma 14 , the example shows that when $\mathcal{B}$ is not reflexive (H2'i) does not follow from Hypothesis 1 .

Example 6. As a further, similar example consider the operator $L: \rho \mapsto-\mathrm{i}[H, \rho]$ defined for $\rho \in \mathcal{J}_{1}(\mathcal{H})=\mathcal{B}$, where $H$ is a bounded self-adjoint operator on the Hilbert space $\mathcal{H}$. Let $H$ have purely continuous spectrum, so that ker $L=\{0\}$. On the other hand, $\operatorname{tr} L \tilde{\rho}=0$ for any $\tilde{\rho} \in \mathcal{J}_{1}(\mathcal{H})$, because $\operatorname{tr} H \tilde{\rho}=\operatorname{tr} \tilde{\rho} H$ ([33], Cor. 3.8). Then $\operatorname{tr} \rho=0$ extends to $\rho \in \overline{\operatorname{ran} L}$, which is thus a proper subspace of $\mathcal{J}_{1}(\mathcal{H})$. 


\section{Applications}

Our results apply to a wide range of driven quantum and classical systems. For quantum systems we consider evolutions generated either by a Hamiltonian or a Lindbladian. We focus on the special class of "dephasing Lindbladians" which are in some sense intermediate between Hamiltonians and generic Lindbladians. As we shall explain, adiabatic evolutions in the Hamiltonian setting have a different character from those in the dephasing setting. In the Hamiltonian case tunneling is reversible while in the dephasing one it is irreversible.

In classical systems we consider continuous-time Markov processes. We give an adiabatic expansion for a slowly driven Markov process with unique stationary distribution and then restrict our attention to reversible processes and to the generation of probability currents.

3.1. Unitary evolutions. The results of Sect. 2 may be applied to recover known facts about the unitary adiabatic evolution driven by a smoothly varying family of self-adjoint Hamiltonians $H(s)$ on a Hilbert space $\mathcal{H}[19,27]$. Consider a simple, discrete eigenvalue $e(s)$ of $H(s)$. Its normalized eigenfunction $\psi(s)$ spans the manifold of instantaneous stationary states, i.e. the kernel of

$$
L(s)=-\mathrm{i}(H(s)-e(s)) .
$$

Equation (1) is the Schrödinger equation and let $\psi_{\varepsilon}(s)$ be its solution with initial data $\psi_{\varepsilon}(0)=\psi(0)$. Tunneling, $T(s)$, is defined as the leaking out from the manifold of stationary states, i.e.

$$
T_{\varepsilon}(s)=1-\left|\left(\psi(s), \psi_{\varepsilon}(s)\right)\right|^{2} .
$$

There is extensive literature (see [15] and references therein) which is concerned with estimates of the tunneling amplitude at all orders in $\varepsilon$, or beyond. The simplest version of these results can be seen to be a consequence of Corollary 7. Namely:

Theorem 15. Suppose that $H(s)=H(s)^{*}$ is a $C^{\infty}$-family in the sense of Definition 1 , and is in addition constant near the endpoints $s=0$ and $s=1$. Let $\psi_{\varepsilon}(s)$ and $\psi(s)$ be as above. Then $T_{\varepsilon}(1)=O\left(\varepsilon^{k}\right)$, for any $k$, see Fig. 2 .

It may be instructive to examine this result from the perspective of the evolution of density matrices generated by the adjoint action of $H$. In contrast with the Schrödinger generator of Eq. (29) whose kernel is one dimensional, the adjoint action $-\mathrm{i}[H(s), \rho]$ has a large kernel spanned by all the stationary states. Tunneling is then described not by the motion in the range but rather by the motion in the kernel. Theorem 15 may then be interpreted as the statement that the adiabatic map is a rigid map of the kernel up to terms of infinite order, at time one, see Fig. 1.

Interestingly, when Theorem 6 is applied to open quantum systems, described by a dephasing Lindbladian one reaches the opposite conclusion, namely that tunneling is irreversible and $O(\varepsilon)$. To complete the picture, it may be worthwhile to discuss from the latter perspective why tunneling gets reduced from first to infinite order in $\varepsilon$ in the special case of the adjoint action. These two points will be addressed in detail in Corollary 19 and thereafter. 
3.2. Evolutions generated by Lindblad operators. Lindbladians arise as generators of dynamical semigroups [13,23]. Different settings are available in the literature. We choose one of them: Let $\mathcal{A}$ be a $C^{*}$-algebra with identity and let $\Phi_{t},(t \geq 0)$ be a normcontinuous semigroup of positive normalized maps on $\mathcal{A}$. As noted in Subsect. 2.2, $\Phi_{t}$ and $\Phi_{t}^{*}$ have norm 1 , and hence are contraction semigroups, with dual generators, $\mathcal{L}_{*}$ and $\mathcal{L}$.

Following [23], $\Phi_{t}$ is called a dynamical semigroup if, besides of the above properties, it is completely positive. We find it convenient to follow the accepted tradition and call the generator in the Schrödinger picture, $\mathcal{L}=\left(\mathcal{L}_{*}\right)^{*}$, the Lindbladian.

More precisely, the generator in the Heisenberg picture $\mathcal{L}_{*}$ is a (weak-* continuous) operator on the Banach space $\mathcal{B}(\mathcal{H})$ of bounded operators on a Hilbert space $\mathcal{H}$. It is thus determined by its restriction on $\mathcal{A}=\operatorname{Com}(\mathcal{H})$, the compact operators on $\mathcal{H}$ (cf. examples of Sect. 2). Then the Lindbladian $\mathcal{L}: \mathcal{J}_{1}(\mathcal{H}) \rightarrow \mathcal{J}_{1}(\mathcal{H})$ has a general form [23]

$$
\mathcal{L} \rho=-\mathrm{i}[H, \rho]+\frac{1}{2} \sum_{\alpha}\left(\left[\Gamma_{\alpha} \rho, \Gamma_{\alpha}^{*}\right]+\left[\Gamma_{\alpha}, \rho \Gamma_{\alpha}^{*}\right]\right)
$$

with $H=H^{*}$ and $\sum_{\alpha} \Gamma_{\alpha}^{*} \Gamma_{\alpha}$ bounded operators on $\mathcal{H}$.

Remark 13. $\mathcal{L}$ does not determine $\Gamma_{\alpha}, H$ uniquely. The "gauge transformation" $\Gamma_{\alpha} \rightarrow$ $\Gamma_{\alpha}+\beta_{\alpha} \mathbb{1}$ and $H \rightarrow H+\mathrm{i} \sum_{\alpha}\left(\beta_{\alpha} \Gamma_{\alpha}^{*}-\overline{\beta_{\alpha}} \Gamma_{\alpha}\right) / 2$ leave $\mathcal{L}$ invariant.

In the generic case the Lindbladian has a 1-dimensional kernel, with $\operatorname{ker} \mathcal{L}_{*}=$ $\operatorname{span}\{\mathbb{1}\}$ independently of $\mathcal{L}$, cf. Lemma 4 . We consider a smoothly varying family of Lindbladians. Let $\rho(s)$ be the corresponding state and $\rho_{\varepsilon}(s)$ be the solution of the adiabatic evolution equation Eq. (1) with initial data $\rho_{\varepsilon}(0)=\rho(0)$. The tunneling Eq. (30) should be generalized to $T=1-F^{2}$, where the fidelity is

$$
F_{\varepsilon}(s)=\operatorname{tr}\left(\left(\rho(s)^{1 / 2} \rho_{\varepsilon}(s) \rho(s)^{1 / 2}\right)^{1 / 2}\right) .
$$

In the presence of a gap a system relaxes to its equilibrium state exponentially fast. A gapped system with a unique ground state will remain close to the instantaneous equilibrium state under adiabatic deformation. For the more interesting, gapless case, see Subsect. 3.6. From the results in the previous section we have:

Theorem 16. Let $\mathcal{L}(s)$ be $C^{\infty}$-family of Lindbladians having a unique instantaneous stationary state $\rho(s)$. Then, by Lemma $2, \rho(s)$ is parallel transported. Suppose that the assumptions of Theorem 6 hold and $L(s)$ is constant near the endpoints $s=0,1$. Then, by Corollary $8, \rho_{\varepsilon}(1)=\rho(1)+O\left(\varepsilon^{k}\right)$ for any $k$ and the tunneling out of the ground state is $T_{\varepsilon}(1)=O\left(\varepsilon^{k}\right)$.

3.3. Dephasing Lindbladians. We say that $\mathcal{L}$ is a dephasing Lindbladian (corresponding to a given Hamiltonian $H$ ) if

$$
\operatorname{ker} \mathcal{L}_{*} \supset \operatorname{ker}([H, \cdot])
$$

as subspaces of $\mathcal{B}(\mathcal{H})$.

By the following proposition, the evolution shares the manifold of stationary states with the corresponding Hamiltonian evolution. 
Proposition 17. In connection with Eq. (31) we have:

1. $\operatorname{ker} \mathcal{L}_{*} \supset \operatorname{ker}([H, \cdot])$ is equivalent to $\Gamma_{\alpha}=f_{\alpha}(H)$ for some functions $f_{\alpha}$.

2. $\Gamma_{\alpha}=f_{\alpha}(H)$ implies $\operatorname{ker} \mathcal{L}=\operatorname{ker}([H, \cdot])$ as subspaces of $\mathcal{J}_{1}(\mathcal{H})$.

3. If the spectrum of $H$ is pure point, then the last implication is an equivalence. This applies in particular to the finite-dimensional case.

A dephasing Lindbladian conserves all observables which are conserved by $H$, in particular $H$ itself and $\Gamma_{\alpha}$. If one interprets the energy of the system in terms of $H$ and $\Gamma$ (see Remark 13) then one learns that although the system is open, it does not exchange energy with a bath. However, the dephasing Lindbladian induces decoherence w.r.t. the energy eigenbasis. A (non-rigorous) scenario where that may arise is discussed in [30].

Example 7. The simplest dephasing Lindbladian is a 2-level system (a qubit). It is a 4-parameter family: The Hamiltonian is determined by the 3-vector $b$

$$
2 H=b \cdot \sigma, \quad\left(b \in \mathbb{R}^{3}, \sigma=\left(\sigma_{1}, \sigma_{2}, \sigma_{3}\right)\right),
$$

where $\sigma_{j}$ are the Pauli matrices and $\gamma \geq 0$ characterizes the dephasing

$$
\mathcal{L} \rho=-\mathrm{i}[H, \rho]+\gamma|b|^{-1}[[H, \rho], H], \quad(\gamma \geq 0) .
$$

(Recall that by $4 H^{2}=(b \cdot b) \mathbb{1}$ any function of $H$ is of the form $f(H)=\alpha H+\beta \mathbb{1}$; the dephasing term is written in such a way that $\gamma$ is dimensionless.) The canonical map of normalized states into the Bloch ball,

$$
\rho \mapsto n \in \mathbb{R}^{3},|n| \leq 1: \quad \rho=\frac{\mathbb{1}+n \cdot \sigma}{2},
$$

maps the evolution equation $\dot{\rho}=\mathcal{L} \rho$ into the Bloch equation [14]

$$
\dot{n}=b \times n+\gamma \hat{b} \times(b \times n),
$$

where $\hat{b}=b /|b|$.

3.4. Adiabatic expansion for dephasing Lindbladians. For simplicity consider $H$ with simple eigenvalues $e_{0}, \ldots, e_{d-1}$ with normalized eigenvectors $\psi_{i}$ :

$$
H=\sum_{i} e_{i} P_{i}, \quad P_{i}=\left|\psi_{i}\right\rangle\left\langle\psi_{i}\right|
$$

on a finite dimensional Hilbert space $\mathcal{H}, \operatorname{dim} \mathcal{H}=d$. The operators $E_{i j}:=\left|\psi_{i}\right\rangle\left\langle\psi_{j}\right|$ form a basis of $\mathcal{B}(\mathcal{H})$, the linear maps on $\mathcal{H}$, which is orthonormal once that space is endowed with the Hilbert-Schmidt inner product. A straightforward computation using Prop. 17 shows that $E_{i j}$ are eigenvectors of $\mathcal{L}$ and the eigenvalues $\mathcal{L} E_{i j}=\lambda_{i j} E_{i j}$ satisfy $\lambda_{i j}=\bar{\lambda}_{j i}, \operatorname{Re} \lambda_{i j} \leq 0$ and $\lambda_{i j}=0$ if and only if $i=j$. Hence ker $\mathcal{L}$ is spanned by $E_{i i}=P_{i}$ and $\operatorname{ran} \mathcal{L}$ by $E_{i j},(i \neq j)$ with the corresponding projections (cf. (11)),

$$
\mathcal{P} \rho=\sum_{i} P_{i} \rho P_{i}, \quad \mathcal{Q} \rho=\sum_{i \neq j} P_{i} \rho P_{j} .
$$

We now consider a smooth family of Lindbladians $\mathcal{L}(s)$ of dephasing form. 
Theorem 18. The equation

$$
\varepsilon \dot{\rho}(s)=\mathcal{L}(s) \rho(s)
$$

admits a solution of the form

$$
\begin{aligned}
\rho(s)= & P_{0}(s)+\varepsilon \sum_{j \neq 0}\left(\frac{P_{j} \dot{P}_{0}}{\lambda_{j 0}}+\frac{\dot{P}_{0} P_{j}}{\lambda_{0 j}}\right) \\
& -\varepsilon \sum_{j \neq 0}\left(P_{0}(s)-P_{j}(s)\right) \int_{0}^{s} \alpha_{j}\left(s^{\prime}\right) d s^{\prime}+O\left(\varepsilon^{2}\right)
\end{aligned}
$$

with

$$
\alpha_{j}(s)=\operatorname{tr}\left(P_{0}(s) \dot{P}_{j}(s)^{2} P_{0}(s)\right) \cdot \frac{\left(-2 \operatorname{Re} \lambda_{0 j}(s)\right)}{\left|\lambda_{0 j}(s)\right|^{2}} \geq 0 .
$$

More generally, the expansion applies to any solution for which it does at $s=0$, e.g. for the one with initial condition $\rho(0)=P_{0}(0)$, if $\dot{P}_{0}(0)=0$.

The expansion (37) is just $\rho(s)=a_{0}(s)+\varepsilon\left(b_{1}(s)+a_{1}(s)\right)+O\left(\varepsilon^{2}\right)$, in this order, with coefficients given in (18-20). Like in the Hamiltonian case, $b_{1}(s) \in \operatorname{ran} \mathcal{Q}(s)$ describes the shift of the slow manifold relative to the manifold of instantaneous stationary states which is reversible in the sense of Corollary 7 . Unlike there, $a_{1}(s) \in \operatorname{ran} \mathcal{P}(s)$ now describes irreversible tunneling by means of a loss and a gain term involving $P_{0}(s)$ and $P_{j}(s),(j \neq 0)$ respectively. More quantitatively, tunneling out of $P_{0}(s)$ is given by Eq. (32) (with $\rho(s)$ there replaced by the rank 1 projection $P_{0}(s)$ ) as

$$
T_{\varepsilon}(s)=1-F_{\varepsilon}^{2}(s)=1-\operatorname{tr}\left(\rho(s) P_{0}(s)\right) .
$$

For arbitrary $\dot{P}_{0}(0)$ we have the following result:

Corollary 19. The solution of $\varepsilon \dot{\rho}(s)=\mathcal{L}(s) \rho(s)$ with the initial condition $\rho(0)=P_{0}(0)$ tunnels like

$$
T_{\varepsilon}(s)=\varepsilon \sum_{j \neq 0} \int_{0}^{s} \alpha_{j}\left(s^{\prime}\right) d s^{\prime}+O\left(\varepsilon^{2}\right)
$$

with $\alpha_{j}(s) \geq 0$ : Tunneling occurs at a non-negative rate, is irreversible and $O(\varepsilon)$.

This result should be contrasted with the small tunneling of infinite order in the unitary case, Theorem 15. Alternatively, that case can be analyzed on the basis of $\mathcal{L}(s) \rho=$ $-\mathrm{i}[H(s), \rho]$, following [27]. The solution clearly remains a projection from $\rho(0)=$ $P_{0}(0)$ on, i.e. $\rho(s)=\rho(s)^{2}$. Using the expansion (14) for $x(s)=\rho(s)$ then yields

$$
a_{n}+b_{n}=\sum_{j=0}^{n}\left(a_{j} a_{n-j}+a_{j} b_{n-j}+b_{j} a_{n-j}+b_{j} b_{n-j}\right) \text {. }
$$

In view of $a_{0}(s)=P_{0}(s)$, see Example 1 , and $b_{0}(s)=0$, this reads $a_{n}=c_{n}+a_{n} P_{0}+P_{0} a_{n}$, where $c_{n}$ depends on $a_{0}, \ldots, a_{n-1}, b_{0} \ldots, b_{n}$. Since $\mathcal{P} a_{n}=a_{n}$ by definition of $a_{n}$, we obtain two recursions for $P_{i} a_{n} P_{i}$, one for $i=0$ and one for $i \neq 0$. Together with Eq. (16), all coefficients $a_{n}(s), b_{n}(s)$ are now determined instantaneously in terms of $H(s)$ and its derivatives, and in particular without reference to the history $H\left(s^{\prime}\right),\left(s^{\prime}<s\right)$. As a result, the tunneling is of infinite order. 


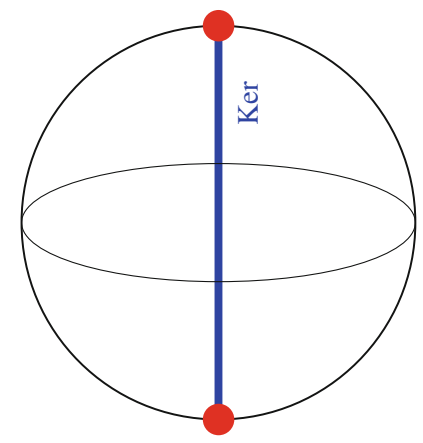

Fig. 4. The states of a qubit (2-level system) can be represented as the 3D ball, the interior of the Bloch sphere. For a dephasing Lindbladian, the set of stationary states is the (blue) axis whose extreme points (red dots) are spectral projections for the Hamiltonian $H$. In the adiabatic setting the (blue) axis moves slowly (color in electronic version only)

Example 8 (continuing Example 7). The adiabatic expansion Eq. (37) takes a rather simple form for the Bloch equation (36). With $\dot{n}$ replaced by $\varepsilon \dot{n}$ and initial condition $n(0)=-\hat{b}(0)$ one finds

$$
n(s)=-\hat{b}(s)+\frac{\varepsilon}{|b(s)|}\left(\frac{\gamma(s) \dot{\hat{b}}(s)+\hat{b}(s) \times \dot{\hat{b}}(s)}{1+\gamma^{2}(s)}+b(s) \int_{0}^{s} \alpha(t) d t\right)+O\left(\varepsilon^{2}\right),
$$

where

$$
\alpha(t)=\frac{\gamma(t)}{1+\gamma^{2}(t)} \frac{|\dot{\hat{b}}(t)|^{2}}{|b(t)|}
$$

The terms in parentheses, in the order as they appear, have the following interpretation: The first term, being proportional to $\gamma \dot{\hat{b}}(s)$ describes friction that causes lagging behind the driver $\hat{b}$. The second term describes "geometric magnetism", a term introduced by [9]. The third term is tunneling and describes motion along the axis towards the center, see Fig. 4. While the first two terms describe instantaneous response in the plane perpendicular to the stationary axis $\hat{b}(s)$, the last term describes irreversible motion inside the Bloch sphere along the axis, Fig. 4.

Proof. That (34) defines the most general dephasing Lindbladian follows from its spectral properties, since the Lindbladian is uniquely determined by the kernel and an offdiagonal eigenvalue $\lambda, \operatorname{Re} \lambda \leq 0$; if 0 stands for the ground state, then $\operatorname{Im} \lambda \geq 0$ for $\lambda=\lambda_{01}$. The Bloch equation follows from the commutation relations $\left[n_{1} \cdot \sigma, n_{2} \cdot \sigma\right]=$ $2 \mathrm{i}\left(n_{1} \times n_{2}\right) \cdot \sigma$. To get the expansion (38) write (37) in the form

$$
\begin{aligned}
\rho(s)= & P_{0}(s)+\frac{\varepsilon}{|\lambda|^{2}}\left(\operatorname{Re} \lambda\left\{P_{1}, \dot{P}_{0}\right\}+\mathrm{i} \operatorname{Im} \lambda\left[P_{1}, \dot{P}_{0}\right]\right) \\
& -\varepsilon\left(P_{0}(s)-P_{1}(s)\right) \int_{0}^{s} \alpha_{1}\left(s^{\prime}\right) d s^{\prime}+O\left(\varepsilon^{2}\right)
\end{aligned}
$$


and use $\lambda=|b|(\mathrm{i}-\gamma)$ as well as the (anti-)commutation relations

$$
\left\{P_{1}, \dot{P}_{0}\right\}=-\frac{1}{2} \dot{\hat{b}} \cdot \sigma, \quad\left[P_{1}, \dot{P}_{0}\right]=-\frac{\mathrm{i}}{2}(\hat{b} \times \dot{\hat{b}}) \cdot \sigma, \quad\left(\dot{P}_{1}\right)^{2}=\frac{|\dot{\hat{b}}|^{2}}{4}
$$

to get the first order correction terms exactly in the same order as they appear in (38).

Solutions of the Bloch equations are illustrated in Fig. 3.

Further applications of driven dephasing Lindbladians are described in [3-5].

3.5. Driven Markov processes. Theorem 6 may be applied to an evolution of the probability distribution of a continuous-time Markov process. In particular, we shall describe below an application to (stochastic) molecular pumps [31] (see also [17,29]).

Let $X$ be a random variable on a finite state space $S=(1,2, \ldots, d)$ and denote

$$
p_{i}=\operatorname{Prob}(X=i) .
$$

The evolution of $X$ is governed by

$$
\dot{p}_{i}=\sum_{j=1}^{d} L_{i j} p_{j}
$$

where the transition rate $j \rightarrow i, L_{i j}(i \neq j)$, is non-negative and $L_{j j}:=-\sum_{i \neq j} L_{i j}$. The transition matrix $\phi(t):=\exp (L t)$ is a left-stochastic matrix $\left(0 \leq \phi_{i j} \leq 1, \sum_{i=1}^{d}\right.$ $\left.\phi_{i j}=1\right)$, a contraction in the norm $\|p\|_{1}=\sum_{j}\left|p_{j}\right|$, and converges to a projection, $\phi(t) \rightarrow P^{+},(t \rightarrow \infty)$ ([34], Thm. 4.4.8). The range of $P^{+}$is spanned by stationary probability distributions, meaning $\sum_{j=1}^{d} L_{i j} \pi_{j}=0$.

We assume that the state space $S$ is indecomposable and denote by $\pi$ the unique stationary distribution of $L$, whence $\operatorname{ker} L=\operatorname{span}\{\pi\}$ and $\operatorname{ran} L=\left\{p \mid \sum p_{i}=0\right\}$. In line with Eq. (13), let $P$ be the rank 1 projection associated to that pair of subspaces, which are left invariant by $L$. We identify $L^{-1}$ with the map $(1-P) L^{-1}(1-P)$ defined on all of $\mathbb{C}^{d}$, and denote its matrix elements by $L_{i j}^{-1}$.

Now we consider a smooth family of generators $L(s)$ with corresponding stationary states $\pi(s)$.

Theorem 20. Assume that $S$ is indecomposable for $L(s)$ and that $\dot{\pi}_{i}(0)=0$. The solution of

$$
\varepsilon \dot{p}_{i}(s)=\sum_{j=1}^{d} L_{i j}(s) p_{j}(s)
$$

with initial condition $p_{i}(0)=\pi_{i}(0)$ is

$$
p_{i}(s)=\pi_{i}(s)+\varepsilon \sum_{j=1}^{d} L_{i j}^{-1}(s) \dot{\pi}_{j}(s)+O\left(\varepsilon^{2}\right) .
$$


Proof. The expansion (41) is just that of Theorem 6. Note that by $\operatorname{ker} P(s)=\operatorname{ran} L(s)$ (or, more abstractly, by Lemma 4 for $\mathcal{A}=\ell^{\infty}(S)$ ) the hypothesis of Corollary 8 is satisfied. Thus $T\left(s, s^{\prime}\right) \pi\left(s^{\prime}\right)=\pi(s)$ and $a_{1}(s)=0$.

We say that $L$ satisfies a detailed balance if

$$
M_{i j}:=L_{i j} \pi_{j}
$$

is a symmetric matrix for some $\pi$, in which case that is the stationary distribution. This can be interpreted as the statement that the current through any link $j \rightarrow i$

$$
J_{i j}(p)=L_{i j} p_{j}-L_{j i} p_{i}
$$

vanishes at equilibrium, $J_{i j}(\pi)=0$.

We now strengthen the assumption on $S$ from indecomposable to irreducible, meaning that $\pi_{j}>0$. Then $\operatorname{ker} M=\operatorname{span}\{(1,1, \ldots 1)\}, \operatorname{ran} M=\operatorname{ran} L$, and the two subspaces decompose $M$ as a linear map. At first $M^{-1}$ is defined on ran $M$, and it may be extended afterwards, arbitrarily but linearly, to all of $\mathbb{C}^{d}$, e.g. by having it vanish on $\operatorname{ker} M$.

In applications to (stochastic) molecular pumps one is interested in systems that carry no current in their equilibrium states, but can be induced to yield net particle transport in an adiabatic pump cycle. Note first that $M$ and $\pi$ provide natural coordinates for those irreducible processes $L$ which satisfy a detailed balance condition. We set the pump period (in scaled time) to be unity.

The net transport across the link $j \rightarrow i$ is expressed in terms of the integrated probability current

$$
T_{i j}:=\frac{1}{\varepsilon} \int_{0}^{1} J_{i j}(p(s)) d s .
$$

The following describes the current in the adiabatic limit.

Corollary 21. Let $s \mapsto\{M(s), \pi(s)\}$ be a pump cycle with $\pi(s)$ the unique equilibrium state for every $s$. Assume that $\dot{\pi}_{j}(0)=0$. Then the transport is geometric to leading order, given by

$$
T_{i j}=\int_{0}^{1} \sum_{k=1}^{d}\left(M_{i j}(s) M_{j k}^{-1}(s)-M_{j i}(s) M_{i k}^{-1}(s)\right) d \pi_{k}(s)+O(\varepsilon) .
$$

In particular $T_{i j}=O(\varepsilon)$ if $\pi$ is constant or, in the periodic case $L(0)=L(1)$, if $M$ is.

Remark 14. Here, geometric means that the transport is independent of the parametrization of the pumping cycle. This is evident in Eq. (44).

Remark 15. The corollary says that effective pump cycles require the variation of both $\pi$ and $M$. As a matter of fact, the long time average of $T_{i j}$ vanishes under the conditions stated in the last line of the corollary regardless of the adiabatic limit [25,26,31].

Proof. The contribution to $T_{i j}$ of order $\varepsilon^{-1}$ vanishes due to the detailed balance condition. To next order Eqs. $(43,41)$ yield

$$
T_{i j}=\int_{0}^{1} \sum_{k=1}^{d}\left(L_{i j}(s) L_{j k}^{-1}(s)-L_{j i}(s) L_{i k}^{-1}(s)\right) \dot{\pi}_{k}(s) d s+O(\varepsilon) .
$$


Equation (42) may be written as $M=(1-P) L(1-P) \Pi$, where $\Pi_{i j}=\pi_{i} \delta_{i j}$, implying $L^{-1}=(1-P) \Pi M^{-1}(1-P)$. Thus, with $P_{j l}=\pi_{j}$, we have

$$
L_{i j} L_{j k}^{-1} \dot{\pi}_{k}=L_{i j} \sum_{l}(1-P)_{j l} \pi_{l} M_{l k}^{-1} \dot{\pi}_{k}=M_{i j} M_{j k}^{-1} \dot{\pi}_{k}-\sum_{l} M_{i j} \pi_{l} M_{l k}^{-1} \dot{\pi}_{k} .
$$

After interchanging $i, j$ and taking the difference, the second term cancels and we are left with (44). The additional claim in the periodic case follows by the fundamental theorem of calculus.

3.6. Remarks about the gapless case. Theorem 11 can be applied to evolutions generated by either a Hamiltonian or a Lindbladian, just like Theorem 6 was in Subsects. 3.1 and 3.2, respectively. The Hilbert space $\mathcal{H}$ must, of course, be infinite-dimensional in order for the gap to vanish.

In the unitary case the result provides a new proof of the adiabatic theorem for Hamiltonians without spectral gap, as in [2,35]; in fact, Hypotheses 1 and 2' are trivially satisfied in this case.

In the Lindbladian case it would be desirable to apply Theorem 11 to the natural space $\mathcal{B}=\mathcal{J}_{1}(\mathcal{H})$. Unfortunately its Hypothesis 2' is typically not satisfied, as Example 6 shows. However, Lindbladians of the dephasing kind have extensions to $\mathcal{J}_{2}(\mathcal{H})$, to which that hypothesis does apply. The result so obtained is applicable to tunneling, as we shall see below. A typical situation leading to a gapless (dephasing) Lindbladian arises from a Hamiltonian having both continuous and point (e.g. discrete) spectrum.

Theorem 22. Let $\mathcal{L}(s), 0 \leq s \leq 1$, be $C^{1}$-family of dephasing Lindbladians acting on $\mathcal{J}_{2}(\mathcal{H})$. Then Eq. (24) holds true. Let $\mathcal{P}(s)$ be the associated projections, for almost all $s$; moreover let $\mathcal{P}(s)$ be defined for all $0 \leq s \leq 1$ and $C^{1}$ as a bounded operator on $\mathcal{J}_{2}(\mathcal{H})$. Then the solution of $\varepsilon \dot{\rho}=\mathcal{L}(s) \rho$ with initial data $\rho(0)=\mathcal{P}(0) \rho(0)$ satisfies

$$
\sup _{s \in[0,1]}\|\rho(s)-\mathcal{T}(s, 0) \rho(0)\|_{\mathcal{J}_{2}(\mathcal{H})} \rightarrow 0, \quad(\varepsilon \rightarrow 0) .
$$

The assumptions on the dephasing Lindbladian $\mathcal{L}(s)$ follow from corresponding ones on the underlying Hamiltonian $H(s)$, see Eq. (33): Let $P_{j}(s)$ be its eigenprojections, for almost all $s$, cf. Remark 9, and let them be defined for all $0 \leq s \leq 1$ and $C^{1}$ uniformly in $j$. Then, as will be proved together with the theorem, $\mathcal{T}(s, 0) P_{j}(0)=P_{j}(s)$. This implies the following result about tunneling out of an initial state given by a rank 1 eigenprojection $\rho(0)=P_{0}(0)$ :

$$
T_{\varepsilon}(s)=1-\operatorname{tr}\left(\rho(s) P_{0}(s)\right)=1-\operatorname{tr}\left(\left(\mathcal{T}(s, 0) P_{0}(0)\right) P_{0}(s)\right)+o(1)=o(1) .
$$

The next example illustrates that, although the tunneling out of the initial state is of order $o(1)$, an adiabatic invariant is conserved up to order $\varepsilon$.

Example 9. Consider the Hamiltonians $H(s)=V(s) H V^{*}(s)$ arising from a $C^{2}$-family of unitaries $V(s)$ and from a bounded $H$. Let $\rho(s)$ solve the equation $\varepsilon \dot{\rho}=-\mathrm{i}[H, \rho]$. Then the energy is an adiabatic invariant in the sense that

$$
|\operatorname{tr}(H(1) \rho(1))-\operatorname{tr}(H(0) \rho(0))|=O(\varepsilon) .
$$

This follows from Eq. (27). We may in fact apply that estimate to $x(s)=\rho(s), \varphi(s)=$ $H(s),\langle\varphi, x\rangle=\operatorname{tr}(H \rho)$ and $\mathcal{L}=-\mathrm{i}[H, \cdot]$, since the assumptions (26) hold true by

$$
\mathcal{L}^{*}(s)(H(s))=0, \quad \dot{H}(s)=-\left[H(s), \dot{V}(s) V^{*}(s)\right]=\mathcal{L}^{*}(s)\left(\mathrm{i} \dot{V}(s) V^{*}(s)\right) .
$$




\section{Proofs and Supplementary Results}

We begin by recalling the Hille-Yosida theorem ([32], Thm. X.47a): A densely defined, closed operator $L$ on $\mathcal{B}$ generates a contraction semigroup iff

$$
\begin{gathered}
(0, \infty) \subset \rho(L), \\
\|(L-\gamma) x\| \geq \gamma\|x\|, \quad(\gamma>0, x \in D(L)) .
\end{gathered}
$$

Conditions (46) reflect the connection between the resolvent and evolution operators. For example the only if part of the Hille-Yosida theorem follows from the formula

$$
-(L-\gamma)^{-1}=\int_{0}^{\infty} \mathrm{e}^{(L-\gamma) t} d t, \quad(\gamma>0) .
$$

Proof of Lemma 1. The hypotheses are a convenient strengthening of those of [32], Thm. X.70, including the remark thereafter. All our statements but uniqueness and Eq. (4) are among its claims, and those two are consequences of its proof. Alternatively, the results may be read off from [20]: Eq. (2) from Thm. 4, Eq. (3) and uniqueness from Thm. 1, and Eq. (4) from Thm. 2.

Proof of Lemma 2. Rank 1 projections $P$ are of the form $P y=\alpha(y) x$, where $x \in \mathcal{B}$ and $\alpha \in \mathcal{B}^{*}$ are determined up to reciprocal factors. Any $\alpha$ with $\operatorname{ker} \alpha=\operatorname{ker} P$ may thus be picked, and then $x$ normalized by $\alpha(x)=1$. Since ker $P(s)$ is independent of $s$, so is our choice of $\alpha$ in $P(s) y=\alpha(y) x(s)$, while $x(s)$ is $C^{1}$. Thus $\dot{P}(s) y=\alpha(y) \dot{x}(s)$, which vanishes for $y \in \operatorname{ker} P(s)$. The claim just proved states $\dot{P}=\dot{P} P$; together with (7) both sides of $P(s)=T\left(s, s^{\prime}\right) P\left(s^{\prime}\right)$ are seen to satisfy the same differential equation in $s$.

Consider the ranges of $P(s)$ and of $Q(s)=\mathbb{1}-P(s)$. As the name suggests, parallel transport $T(s, 0)$ is obtained by projecting vectors from either subspace at 0 to the corresponding one at $s$ or, more precisely, by repeating the procedure on the intervals of an ever finer partition of $[0, s]$. In fact,

$$
P(s) P(0)+Q(s) Q(0)=\mathbb{1}+[\dot{P}(0), P(0)] s+o(s), \quad(s \rightarrow 0),
$$

implying by Eq. (5),

$$
\begin{aligned}
T\left(s, s^{\prime}\right) & =\lim _{N \rightarrow \infty} \prod_{i=0}^{N-1}\left(P\left(s_{i+1}\right) P\left(s_{i}\right)+Q\left(s_{i+1}\right) Q\left(s_{i}\right)\right) \\
& =\lim _{N \rightarrow \infty}\left(\prod_{i=0}^{N} P\left(s_{i}\right)+\prod_{i=0}^{N} Q\left(s_{i}\right)\right),
\end{aligned}
$$

where $s^{\prime}=s_{0} \leq s_{1} \leq \cdots \leq s_{N}=s$ is a partition of $\left[s^{\prime}, s\right]$ into intervals of length $\left|s_{i+1}-s_{i}\right|=N^{-1}\left|s-s^{\prime}\right|$ and $\prod_{i=0}^{N-1} A_{i}=A_{N-1} \cdots A_{0}$.

Proof of Proposition 3. Since the product of state preserving maps is state preserving the first claim follows from (47). As a result $\mathcal{T}\left(s, s^{\prime}\right) \mathcal{P}\left(s^{\prime}\right)$ maps $\mathcal{S}\left(s^{\prime}\right) \rightarrow \mathcal{S}(s)$, with inverse $\mathcal{T}\left(s^{\prime}, s\right) \mathcal{P}(s)$. The first bullet follows from Eq. (10) for the two maps, i.e.

$$
\left\|\rho\left(s^{\prime}\right)-\tilde{\rho}\left(s^{\prime}\right)\right\|=\left\|\mathcal{T}\left(s^{\prime}, s\right) \mathcal{P}(s)(\rho(s)-\tilde{\rho}(s))\right\| \leq\|\rho(s)-\tilde{\rho}(s)\| .
$$


Any convex decomposition of $\mathcal{T}\left(s, s^{\prime}\right) \rho\left(s^{\prime}\right),\left(\rho\left(s^{\prime}\right) \in \mathcal{S}\left(s^{\prime}\right)\right)$ entails one of $\rho\left(s^{\prime}\right)$, which yields the second bullet in the variant where the bracketed word is omitted. The continuity of $\mathcal{T}\left(s, s^{\prime}\right) \rho$ w.r.t. $\rho$ yields the other variant. To obtain the last statement we note that $\mathcal{T}\left(s, s^{\prime}\right) \rho\left(s^{\prime}\right)$ is, for fixed $s$ and all $s^{\prime}$, an isolated extreme point in $\mathcal{S}(s)$, just like $\rho(s)$. They agree, since they do for $s^{\prime}=s$.

Proof of Lemma 4. By the setting of Subsect. 2.2, $\mathcal{P}$ has a predual $\mathcal{P}_{*}$, which is also of rank 1 . Since $\mathcal{P}_{*}(\mathbb{1})=\mathbb{1}$ by the normalization condition, we have $\operatorname{ran} \mathcal{P}_{*}=\operatorname{span}\{\mathbb{1}\}$. Thus ker $\mathcal{P}=\left(\operatorname{ran} \mathcal{P}_{*}\right)^{\perp}$ is independent of $\mathcal{P}$. We recall that $S^{\perp} \subset \mathcal{B}=\mathcal{A}^{*}$ is the annihilator of a subspace $S \subset \mathcal{A}$. The remaining claims follow from Lemma 2 and Proposition 3.

Proof of Proposition 5. $L a=0$ and $a=L b$ imply $(L-\gamma)(a+\gamma b)=-\gamma^{2} b$ and by (46) $\gamma\|a+\gamma b\| \leq \gamma^{2}\|b\|$ for $\gamma>0$. After dividing by $\gamma$ we obtain $a=0$ in the limit $\gamma \rightarrow 0$.

Before proving Theorem 6 we derive a few further consequences of its assumptions. Hypothesis 2 includes Eq. (12); moreover the pair of subspaces decomposes $L$. The restriction $L \uparrow \operatorname{ran} L$ is closed and has $\operatorname{range} \operatorname{ran}(L \uparrow \operatorname{ran} L)=\operatorname{ran} L$; by (12) it is one-to-one. Thus $0 \notin \sigma(L \uparrow \operatorname{ran} L)$. Together with $\sigma(L \uparrow \operatorname{ker} L) \subset\{0\}$ we conclude that the resolvent set contains a punctured neighborhood of 0 , which proves the presence of a gap.

We maintain that the projection $P$ is given by the Riesz projection, see Eq. (28) with $\lambda=0$. Calling the latter temporarily $\tilde{P}$ we thus claim $\tilde{P} a=a$ for $a \in \operatorname{ker} L$ and $\tilde{P} b=0$ for $b \in \operatorname{ran} L$. The first statement is evident from (28); for the second it suffices, by $\tilde{P} L \subset L \tilde{P}$, to show that $\operatorname{ran} \tilde{P} \cap \operatorname{ran} L=\{0\}$. This in turn follows because $L \uparrow(\operatorname{ran} \tilde{P} \cap \operatorname{ran} L)$ is a bounded operator with empty spectrum; in fact, it is contained in $\sigma(L \uparrow \operatorname{ran} \tilde{P}) \cap \sigma(L \uparrow \operatorname{ran} L)=\varnothing$ since the first spectrum is contained in $\{0\}$, while the second is disjoint from it. Finally, we recall the formula for the inverse of $L \uparrow \operatorname{ran}(1-P)$ ([21], Eq. (III.6.23)):

$$
L^{-1}=-\frac{1}{2 \pi \mathrm{i}} \oint_{\Gamma}(L-z)^{-1} \frac{d z}{z} .
$$

In particular, $P(s)$ and $L(s)^{-1}$ are $C^{k}$ in norm.

Proof of Theorem 6. We insert the right-hand side of (14) as an ansatz into (1) and equate orders $\varepsilon^{n},(n=0, \ldots, N)$, resp. $O\left(\varepsilon^{N+1}\right)$. We find

$$
\begin{aligned}
L b_{0} & =0 \\
\dot{a}_{n}+\dot{b}_{n} & =L b_{n+1}, \quad(n=0, \ldots, N-1), \\
\varepsilon \dot{r}_{N}+\dot{a}_{N}+\dot{b}_{N} & =L r_{N} .
\end{aligned}
$$

In particular, $b_{0}=0$. Note that $Q a=0$ implies $\dot{Q} a+Q \dot{a}=0$, or $Q \dot{a}=-\dot{Q} a=\dot{P} a$. Similarly, $P \dot{b}=-\dot{P} b$. Applying $Q$ and $P$ to (48) yields

$$
\begin{aligned}
& \dot{P} a_{n}+Q \dot{b}_{n}=L b_{n+1}, \\
& P \dot{a}_{n}-\dot{P} b_{n}=0 .
\end{aligned}
$$

If $b_{n}$ is known, (51) implies

$$
\dot{a}_{n}=Q \dot{a}_{n}+P \dot{a}_{n}=\dot{P} a_{n}+\dot{P} b_{n},
$$


the solution of which is (15) by Eq. (7) and the Duhamel formula. If $a_{n}$ and $b_{n}$ are known, $b_{n+1}$ follows from (50) and Lemma 13, provided $b_{n}$ is differentiable (see below). All this determines $b_{0}, a_{0}, b_{1}, \ldots, a_{N-1}, b_{N}$. We then define $a_{N}, b_{N+1}$ by the same Eqs. (15, 16), which ensures $\dot{a}_{N}+\dot{b}_{N}=L b_{N+1}$. Then (49) reads

$$
\varepsilon \dot{r}_{N}=L r_{N}-L b_{N+1}
$$

with solution

$$
\begin{aligned}
r_{N}(\varepsilon, s) & =U_{\varepsilon}(s, 0) r_{N}(\varepsilon, 0)-\varepsilon^{-1} \int_{0}^{s} U_{\varepsilon}\left(s, s^{\prime}\right) L\left(s^{\prime}\right) b_{N+1}\left(s^{\prime}\right) d s^{\prime} \\
& =U_{\varepsilon}(s, 0) r_{N}(\varepsilon, 0)+\int_{0}^{s}\left(\frac{\partial}{\partial s^{\prime}} U_{\varepsilon}\left(s, s^{\prime}\right)\right) b_{N+1}\left(s^{\prime}\right) d s^{\prime} .
\end{aligned}
$$

An integration by parts yields (17) and the bound on the remainder follows from Hypothesis 1 through Eq. (3). Inspection of the recursion relations shows $a_{n}, b_{n} \in C^{N+2-n}$, which provides the required differentiability.

Proof of Theorem 9. For an adiabatic invariant $\varphi$ Eq. (23) together with $\langle\varphi(0), x(0)\rangle=$ $\langle\varphi(s), T(s, 0) x(0)\rangle$ yields

$$
|\langle\varphi(s), x(s)-T(s, 0) x(0)\rangle| \leq C \varepsilon\|\varphi(s)\|\|x(0)\| .
$$

The claim follows from $\|P(s) x\|=\sup \left\{|\langle\varphi, x\rangle| \mid \varphi \in \operatorname{ker} L^{*}(s),\|\varphi\|=1\right\}$.

Proof of Proposition 10. The assumption (21) may be phrased differently. The projections $P^{*}$ and $Q^{*}$ are associated to ker $L^{*} \oplus$ ran $L^{*}$, because the subspaces (13) decompose $L$. Thus $Q^{*} \varphi=0, P^{*} \dot{\varphi}=0$ just means that $\varphi(s) \in$ ran $P^{*}(s)$ is parallel transported: $\varphi(s)=T^{*}\left(s, s^{\prime}\right) \varphi\left(s^{\prime}\right)$.

Equation (22) follows from

$$
\begin{aligned}
\frac{d}{d s}\langle\varphi, x\rangle & =\langle\dot{\varphi}, x\rangle+\langle\varphi, \dot{x}\rangle=\left\langle L^{*} L^{*-1} \dot{\varphi}, x\right\rangle+\varepsilon^{-1}\langle\varphi, L x\rangle \\
& =\left\langle L^{*-1} \dot{\varphi}, L x\right\rangle+\varepsilon^{-1}\left\langle L^{*} \varphi, x\right\rangle=\varepsilon\left\langle L^{*-1} \dot{\varphi}, \dot{x}\right\rangle+0 .
\end{aligned}
$$

Integration by parts in (22) gives

$$
\left.\langle\varphi, x\rangle\right|_{0} ^{s}=\varepsilon\left(\left.\langle\phi, x\rangle\right|_{0} ^{s}-\int_{0}^{s}\left\langle\dot{\phi}\left(s^{\prime}\right), x\left(s^{\prime}\right)\right\rangle d s^{\prime}\right),
$$

where $\phi\left(s^{\prime}\right)=L^{*}\left(s^{\prime}\right)^{-1} \dot{\varphi}\left(s^{\prime}\right)$. We observe that $\left\|x\left(s^{\prime}\right)\right\| \leq\|x(0)\|$ by Lemma 1 , and $\dot{\varphi}(s)=\dot{P}^{*}(s) \varphi(s)$ by (7). By (9) we see that $\left\|\varphi\left(s^{\prime}\right)\right\|,\left\|\phi\left(s^{\prime}\right)\right\|$ and $\left\|\dot{\phi}\left(s^{\prime}\right)\right\|$ are bounded by a constant times $\|\varphi(s)\|$, proving Eq. (23).

We now turn to the case without gap.

Proof of Theorem 11. Let us first dispose of the "almost all" qualifier in the theorem. We observe that, by continuity, $L(s) P(s)=0$ holds for all $0 \leq s \leq 1$. In particular, $L(s) x_{0}(s)=0$ for $x_{0}(s)=T(s, 0) x(0)$. The remainder to be estimated is $r(s)=$ $x(s)-x_{0}(s)$. By Eq. (1) it satisfies the differential equation $\varepsilon \dot{r}(s)=L(s) r(s)-\varepsilon \dot{x}_{0}(s)$ with solution

$$
r(s)=-\int_{0}^{s} U_{\varepsilon}\left(s, s^{\prime}\right) \dot{x}_{0}\left(s^{\prime}\right) d s^{\prime}
$$


By Eqs. $(6,24)$ we have $\dot{x}_{0}(s) \in \overline{\operatorname{ran} L(s)}$ for almost all $s$. This property is reflected in the splitting

$$
\dot{x}_{0}(s)=L(s)(L(s)-\gamma)^{-1} \dot{x}_{0}(s)-\gamma(L(s)-\gamma)^{-1} \dot{x}_{0}(s), \quad(\gamma>0),
$$

motivated by Eq. (53) below. Together they yield

$$
\begin{aligned}
r(s)= & -\int_{0}^{s} U_{\varepsilon}\left(s, s^{\prime}\right) L\left(s^{\prime}\right)\left(L\left(s^{\prime}\right)-\gamma\right)^{-1} \dot{x}_{0}\left(s^{\prime}\right) d s^{\prime} \\
& +\int_{0}^{s} U_{\varepsilon}\left(s, s^{\prime}\right) \gamma\left(L\left(s^{\prime}\right)-\gamma\right)^{-1} \dot{x}_{0}\left(s^{\prime}\right) d s^{\prime},
\end{aligned}
$$

where, by an appropriate choice of $\gamma>0$, the second integral can be made arbitrarily small by means of dominated convergence; in fact, uniformly in $\varepsilon$ due to $\left\|U_{\varepsilon}\left(s, s^{\prime}\right)\right\| \leq 1$. It remains to show that, for fixed $\gamma>0$, the first integral vanishes with $\varepsilon$. To illustrate the argument, let us temporarily pretend that $z(s):=(L(s)-\gamma)^{-1} \dot{x}_{0}(s)$ is $C^{1}$. Since $\varepsilon \partial_{s^{\prime}} U_{\varepsilon}\left(s, s^{\prime}\right)=-U_{\varepsilon}\left(s, s^{\prime}\right) L\left(s^{\prime}\right)$ an integration by parts yields for that integral

$$
\varepsilon \int_{0}^{s} \partial_{s^{\prime}} U_{\varepsilon}\left(s, s^{\prime}\right) z\left(s^{\prime}\right) d s^{\prime}=\left.\varepsilon U_{\varepsilon}\left(s, s^{\prime}\right) z\left(s^{\prime}\right)\right|_{s^{\prime}=0} ^{s^{\prime}=s}-\varepsilon \int_{0}^{s} U_{\varepsilon}\left(s, s^{\prime}\right) \frac{d}{d s^{\prime}} z\left(s^{\prime}\right) d s^{\prime},
$$

and exhibits the desired property for $\varepsilon \rightarrow 0$. Finally, we get rid of the additional assumption by amending the argument as follows. We introduce a mollifier $j,(j \in$ $\left.C_{0}^{\infty}(\mathbb{R}), \int j(x) d x=1\right)$ and set $j_{\delta}(x)=\delta^{-1} j(x / \delta),(\delta>0)$; we extend $\dot{x}_{0}$ continuously outside of the interval $[0,1]$; and split

$$
z=(L-\gamma)^{-1}\left(\dot{x}_{0}-j_{\delta} * \dot{x}_{0}\right)+(L-\gamma)^{-1}\left(j_{\delta} * \dot{x}_{0}\right) .
$$

Since $\dot{x}_{0}-j_{\delta} * \dot{x}_{0} \rightarrow 0,(\delta \rightarrow 0)$ and $\left\|L(L-\gamma)^{-1}\right\|$ is bounded, both uniformly in $s$, the first term contributes arbitrary little to the integral, uniformly in $\varepsilon$, if $\delta$ is picked small enough. The preliminary argument can now be applied to the second term in place of $z$.

Proof of Proposition 12. Equation (52) can be obtained from the present assumptions by replacing $\phi$ for $L^{*-1} \dot{\varphi}$ in the previous derivation.

Proof of Lemma 13. Suppose a closed operator $L$ has 0 as an isolated point in its spectrum, with associated Riesz projection $P$, see Eq. (28) with $\lambda=0$. Then $P$ decomposes $L$ with $\sigma(L \uparrow \operatorname{ran} P)=\{0\}$ and $\sigma(L \uparrow \operatorname{ran}(1-P))=\sigma(L) \backslash\{0\}$ ([21], Thm. III.6.17), whence $\operatorname{ran} L \supset \operatorname{ran}(1-P)$ and $\operatorname{ker} L \subset \operatorname{ran} P$. As a result, (H2i) implies that $L$ is Fredholm because both its parts are, and in particular that (H2ii) holds, regardless of Hypothesis 1.

From now on we assume (H1) and (H2ii), with the former implying Eqs. $(46,12)$. By the stability theorem ([21], Thm. IV.5.31) $L-z$ remains semi-Fredholm for $z$ in a complex neighborhood of 0 and the index $\operatorname{dim} \operatorname{ker}(L-z)-\operatorname{dim}(\mathcal{B} / \operatorname{ran}(L-z))$ is constant; moreover the two dimensions are separately constant in a punctured neighborhood $U$. By (46), they both vanish there, and so does the index at $z=0$. This has the following implications: First, if $0 \in \sigma(L)$, then it is isolated. Second, the map $\operatorname{ker} L \rightarrow \mathcal{B} / \operatorname{ran} L, a \mapsto a+\operatorname{ran} L$ is one-to-one by (12) and thus onto by the vanishing index. This proves $\operatorname{ker} L+\operatorname{ran} L=\mathcal{B}$, completing the proof of Eq. (13).

Finally in order to prove $(\mathrm{H} 2 \mathrm{i})$, we observe that the Riesz projection is given by $P$, as established at the beginning of this section. Thus it is finite-dimensional because $\operatorname{ker} L$ is. 
Lemma 14 is an immediate consequence of the last two statements of the following lemma.

Lemma 23. Let $L$ be the generator of a contraction semigroup on $\mathcal{B}$. Then

$$
\begin{gathered}
b \in \overline{\operatorname{ran} L} \Leftrightarrow \lim _{\gamma \downarrow 0} \gamma(L-\gamma)^{-1} b=0, \quad(b \in \mathcal{B}), \\
\operatorname{ker} L \cap \overline{\operatorname{ran} L}=\{0\} .
\end{gathered}
$$

If, in addition, $\mathcal{B}$ is reflexive, then $\overline{\operatorname{ker} L+\operatorname{ran} L}=\mathcal{B}$.

Proof. We note that $\gamma(L-\gamma)^{-1}$ is uniformly bounded in $\gamma>0$ by (46). By density, it thus suffices to prove the direct implication (53) for $b=L x$, for which it follows from $\gamma(L-\gamma)^{-1} L x=\gamma\left(x+\gamma(L-\gamma)^{-1} x\right)$. Conversely, set $x_{\gamma}=(L-\gamma)^{-1} b$; then $L x_{\gamma}=b+\gamma(L-\gamma)^{-1} b \rightarrow b$.

Next, let $b$ be in the intersection (54): we have $(L-\gamma) b=-\gamma b$, and hence $b=$ $-\gamma(L-\gamma)^{-1} b$, which vanishes as $\gamma \downarrow 0$.

To prove the last claim it suffices, by the Hahn-Banach theorem, to show that $x^{*} \in$ $(\operatorname{ker} L)^{\perp} \cap(\operatorname{ran} L)^{\perp}$ implies $x^{*}=0$. Here $S^{\perp} \subset \mathcal{B}^{*}$ is the annihilator of a subspace $S \subset \mathcal{B}$. We have $(\operatorname{ran} L)^{\perp}=\operatorname{ker} L^{*}$ and, in the reflexive case, $(\operatorname{ker} L)^{\perp}=\overline{\operatorname{ran} L^{*}}$. The last equality is due to $S^{\perp \perp}=\bar{S}$ ([21], Eq. III.1.24). The property $(0, \infty) \subset \rho(L)$ and the uniform bound on $\gamma(L-\gamma)^{-1},(\gamma>0)$ are inherited by $L^{*}$, and so is the consequence (54). We conclude that $x^{*}=0$. (As a matter of fact, $L^{*}$ is also densely defined ([21] Thm. III.5.29) by reflexivity, and hence is the generator of a contraction semigroup; in particular $\left(\mathrm{e}^{L t}\right)^{*}=\mathrm{e}^{L^{*} t}$.)

This concludes the proofs of Sect. 2 and we pass to those of Sect. 3. Actually, Theorems 15 and 16 do not require proof, as they are immediate applications of Sect. 2, while the results of Subsect. 3.5 are proven there.

Proof of Proposition 17. To begin we write Eq. (31) as

$$
\mathcal{L} \rho=-\mathrm{i}[H, \rho]+\frac{1}{2} \sum_{\alpha}\left(2 \Gamma_{\alpha} \rho \Gamma_{\alpha}^{*}-\left\{\rho, \Gamma_{\alpha}^{*} \Gamma_{\alpha}\right\}\right),
$$

and hence

$$
\mathcal{L}_{*} a=\mathrm{i}[H, a]+\frac{1}{2} \sum_{\alpha}\left(2 \Gamma_{\alpha}^{*} a \Gamma_{\alpha}-\left\{a, \Gamma_{\alpha}^{*} \Gamma_{\alpha}\right\}\right) .
$$

It is evident that $\Gamma_{\alpha}=f_{\alpha}(H)$ implies $\operatorname{ker} \mathcal{L}_{*} \supset \operatorname{ker}([H, \cdot])$ and, through $\left[\Gamma_{\alpha}^{*}, \Gamma_{\alpha}\right]=0$, also $\operatorname{ker} \mathcal{L} \supset \operatorname{ker}([H, \cdot])$. Together with a detail to be supplied momentarily, the three claims thus reduce to the following ones:

1. $\operatorname{ker} \mathcal{L}_{*} \supset \operatorname{ker}([H, \cdot])$ implies $\Gamma_{\alpha} \in\{f(H)\}^{\prime \prime} \equiv\{f(H) \mid f(H) \in \mathcal{B}(\mathcal{H})\}^{\prime \prime}$.

2. $\Gamma_{\alpha}=f_{\alpha}(H)$ implies $\operatorname{ker} \mathcal{L} \subset \operatorname{ker}([H, \cdot])$.

3. If the spectrum of $H$ is pure point and if $\operatorname{ker} \mathcal{L} \supset \operatorname{ker}([H, \cdot])$, then $\Gamma_{\alpha} \in\{f(H)\}^{\prime \prime}$.

Here a prime denotes the commutant. We recall ([11], Thm. 2.4.11) that the bicommutant is the strong closure of the original $*$-algebra. Note that $\{f(H)\}$ is strongly closed.

The implications 1-3 are based on the readily verified identity [23]

$$
\mathcal{L}_{*}\left(a^{*} a\right)-a^{*} \mathcal{L}_{*}(a)-\mathcal{L}_{*}\left(a^{*}\right) a=\sum_{\alpha}\left[a, \Gamma_{\alpha}\right]^{*}\left[a, \Gamma_{\alpha}\right] .
$$


1. Let $a \in \operatorname{ker}([H, \cdot])=\{f(H)\}^{\prime}$. Since that subspace of $\mathcal{B}(\mathcal{H})$ is closed under taking adjoints and products, each term in the 1.h.s. of (55) vanishes by the assumption of the present item, implying $\Gamma_{\alpha} \in\{f(H)\}^{\prime \prime}$.

2. Under the assumption, $\mathcal{L}$ acts as $\mathcal{L}_{*}$ under the replacement $H \rightarrow-H, \Gamma_{\alpha} \rightarrow \Gamma_{\alpha}^{*}$. Since $\operatorname{tr} \mathcal{L}(\rho)=0$ for $\rho \in \mathcal{J}_{1}(\mathcal{H})$, Eq. (55) implies

$$
-\operatorname{tr} \rho^{*} \mathcal{L}(\rho)-\operatorname{tr} \rho \mathcal{L}\left(\rho^{*}\right)=\sum_{\alpha} \operatorname{tr}\left[\rho, \Gamma_{\alpha}^{*}\right]^{*}\left[\rho, \Gamma_{\alpha}^{*}\right] .
$$

Thus $\rho \in \operatorname{ker} \mathcal{L}$ implies $\left[\rho, \Gamma_{\alpha}^{*}\right]=0$ and, by $\mathcal{L}\left(\rho^{*}\right)=\mathcal{L}(\rho)^{*}$, also $\left[\rho, \Gamma_{\alpha}\right]=0$. We conclude $[H, \rho]=0$.

3. By the first assumption we can pick finite-rank projections $P_{n}$, which are sums of eigenprojections of $H$ or of subprojections thereof, such that $P_{n} \stackrel{s}{\rightarrow} 1$. In particular $\left[H, P_{n}\right]=0$.

If $a \in \mathcal{J}_{1}(\mathcal{H})$ then $\mathcal{L}_{*}(a) \in \mathcal{J}_{1}(\mathcal{H})$ and, we claim, $\operatorname{tr} \mathcal{L}_{*}(a)=0$ by our second assumption. Indeed, it implies $\mathcal{L}\left(P_{n}\right)=0$ and hence

$$
\operatorname{tr} \mathcal{L}_{*}(a)=\lim _{n} \operatorname{tr}\left(\mathcal{L}_{*}(a) P_{n}\right)=\lim _{n} \operatorname{tr}\left(a \mathcal{L}\left(P_{n}\right)\right)=0 .
$$

Let now $a \in \mathcal{J}_{1}(\mathcal{H}) \cap\{f(H)\}^{\prime}$. Then $\mathcal{L}(a)=0$ and $\operatorname{tr}\left(\mathcal{L}_{*}\left(a^{*}\right) a\right)=\operatorname{tr}\left(a^{*} \mathcal{L}(a)\right)=0$. By taking the trace of Eq. (55) we conclude $\left[a, \Gamma_{\alpha}\right]=0$. The conclusion extends to $a \in\{f(H)\}^{\prime}$ since $P_{n} a \stackrel{s}{\rightarrow} a$. This proves the claim.

Proof of Theorem 18. Clearly,

$$
\begin{aligned}
\dot{\mathcal{P}} \rho & =\sum_{i}\left(\dot{P}_{i} \rho P_{i}+P_{i} \rho \dot{P}_{i}\right), \\
\mathcal{L}^{-1} E_{i j} & =\lambda_{i j}^{-1} E_{i j}, \quad(i \neq j) .
\end{aligned}
$$

We note that

$$
\mathcal{T}\left(s, s^{\prime}\right) P_{k}\left(s^{\prime}\right)=P_{k}(s) .
$$

In fact, the 1.h.s. satisfies the differential equation (5), viz.

$$
\begin{aligned}
\frac{d}{d s} \rho(s) & =\dot{\mathcal{P}}(s) \rho(s), \\
\rho\left(s^{\prime}\right) & =P_{k}\left(s^{\prime}\right),
\end{aligned}
$$

and so does the r.h.s., since

$$
\dot{\mathcal{P}} P_{k}=\sum_{i} \dot{P}_{i} P_{k} P_{i}+P_{i} P_{k} \dot{P}_{i}=\dot{P}_{k} P_{k}+P_{k} \dot{P}_{k}=\dot{P}_{k} .
$$

The claim now follows from (18-20) with $a_{0}(0)=P_{0}(0)$. Indeed, the middle term of (37) follows from (19) and (56):

$$
\begin{aligned}
\mathcal{L}^{-1} \dot{\mathcal{P}} P_{0} & =\mathcal{L}^{-1} \dot{P}_{0}=\mathcal{L}^{-1} \sum_{j \neq 0} P_{j} \dot{P}_{0} P_{0}+P_{0} \dot{P}_{0} P_{j} \\
& =\sum_{j \neq 0} \lambda_{j 0}^{-1} P_{j} \dot{P}_{0} P_{0}+\lambda_{0 j}^{-1} P_{0} \dot{P}_{0} P_{j}=\sum_{j \neq 0} \lambda_{j 0}^{-1} P_{j} \dot{P}_{0}+\lambda_{0 j}^{-1} \dot{P}_{0} P_{j}
\end{aligned}
$$


For the last term of (37) we compute with (58)

$$
\begin{aligned}
\dot{\mathcal{P}} \mathcal{L}^{-1} \dot{\mathcal{P}} P_{0} & =\sum_{j \neq 0} \dot{\mathcal{P}}\left(\lambda_{j 0}^{-1} P_{j} \dot{P}_{0} P_{0}+\lambda_{0 j}^{-1} P_{0} \dot{P}_{0} P_{j}\right) \\
& =\sum_{j \neq 0}\left(\lambda_{j 0}^{-1}+\lambda_{0 j}^{-1}\right)\left(P_{0} \dot{P}_{j}^{2} P_{0}-P_{j} \dot{P}_{0}^{2} P_{j}\right) \\
& =\sum_{j \neq 0} \alpha_{j}\left(P_{j}-P_{0}\right),
\end{aligned}
$$

(with termwise equality) where we have used $\dot{P}_{i} P_{k}=-P_{i} \dot{P}_{k}$ and $\operatorname{tr}\left(P_{j} \dot{P}_{0}^{2} P_{j}\right)=$ $\operatorname{tr}\left(P_{0} \dot{P}_{j}^{2} P_{0}\right)$. Together with (20) the expansion follows. The generalization follows because of the contraction property of the propagator, Eq. (3).

Proof of Corollary 19. The statement evidently applies to the "slow manifold" solution (37), which however does not satisfy $\rho(0)=P_{0}(0)$, as required for the present solution. We compare the two by means of Remark 7. For their difference $\tilde{\rho}(s)$ we have $\|\tilde{\rho}(0)\|=O(\varepsilon)$ implying $\|\mathcal{P}(s) \tilde{\rho}(s)\|=O\left(\varepsilon^{2}\right)$.

Proof of Theorem 22. As noted at the beginning of Subsec. 3.2 a Lindbladian $\mathcal{L}$ is the generator of a contraction on $\mathcal{J}_{1}(\mathcal{H})$, while $\mathcal{L}_{*}$ is on $\operatorname{Com}(\mathcal{H})$; for a dephasing Lindbladian the latter also applies to $\mathcal{L}$ itself, as noted in the proof of Proposition 17. Then, by interpolation, $\mathcal{L}$ generates a contraction also on $\mathcal{J}_{2}(\mathcal{H})$.

Given that $\mathcal{J}_{2}(\mathcal{H})$ is reflexive, Eq. (24) follows from Lemma 14 by showing that ker $L+\overline{\operatorname{ran} L}$ is closed. We will actually show that the two subspaces are orthogonal w.r.t. to the inner product $\langle\cdot, \cdot\rangle$ of $\mathcal{J}_{2}(\mathcal{H})$. In fact, for a dephasing Lindbladian the statement $\operatorname{ker} \mathcal{L}=\operatorname{ker}([H, \cdot])$ from Prop. 17 also holds true as subspaces of $\mathcal{J}_{2}(\mathcal{H})$, as inspection of the proof shows. Thus $\operatorname{ker} \mathcal{L} \subset \operatorname{ker} \mathcal{L}_{*}$ by (33). Then $\mathcal{L} a=0$ and $b=\mathcal{L} \tilde{b}$ imply $\langle a, b\rangle=\left\langle\mathcal{L}_{*} a, \tilde{b}\right\rangle=0$. The theorem follows.

To prove the statements following the theorem let us note that, for almost all $s$,

$$
\mathcal{P}(s) \rho=\sum_{j} P_{j}(s) \rho P_{j}(s),
$$

where the sum runs over the eigenvalues of $H(s)$. In fact, this follows by the RAGE theorem ([12], Thm. 5.8), i.e.

$$
\frac{1}{T} \int_{0}^{T} \mathrm{e}^{\mathrm{i} H t} \rho \mathrm{e}^{-\mathrm{i} H t} d t \rightarrow \sum_{j} P_{j} \rho P_{j}, \quad(T \rightarrow \infty)
$$

for $\rho \in \operatorname{Com}(\mathcal{H})$. It implies $\rho=\sum_{j} P_{j} \rho P_{j}$ for $\rho \in \operatorname{ker} \mathcal{L}$. The converse is obvious. At this point the regularity of $\mathcal{P}(s)$ is inherited from that of the $P_{j}(s)$, and Eq. (59) extends to all $s$. Finally, $\mathcal{T}(s, 0) P_{j}(0)=P_{j}(s)$ follows like $(57)$.

Acknowledgements. Example 5 is due to Gang Zhou. The results of Subsec. 3.5 were derived in collaboration with Nicholas Crawford. We thank both of them for their contribution and for useful discussions. JEA is supported by the ISF and the fund for promotion of research at the Technion. MF is supported by the UNESCO. The authors benefitted from mutual visits supported by their coauthors' institutions. 


\section{References}

1. Abou Salem, W.K.: On the quasi-static evolution of nonequilibrium steady states. Ann. H. Poincaré 8, 569596 (2007)

2. Avron, J.E., Elgart, A.: Adiabatic theorem without a gap condition. Commun. Math. Phys. 203, 445463 (1999)

3. Avron, J.E., Fraas, M., Graf, G.M., Grech, P.: Landau-Zener tunneling for dephasing lindblad evolutions. Commun. Math. Phys. 305(3), 633-639 (2011)

4. Avron, J.E., Fraas, M., Graf, G.M., Grech, P.: Optimal time-schedule for adiabatic evolution. Phys. Rev. A 82, 040304 (2010)

5. Avron, J.E., Fraas, M., Graf, G.M., Kenneth, O.: Geometry of quantum transport for dephasing lindbladians. New J. Phys. 13, 053042 (2011)

6. Avron, J.E., Seiler, R., Yaffe, L.G.: Adiabatic theorems and applications to the quantum Hall effect. Commun. Math. Phys. 110, 33-49 (1987)

7. Berry, M.V.: Quantal phase factors accompanying adiabatic changes. Proc. R. Soc. Lond. A 392, 45-57 (1984)

8. Berry, M.V.: Histories of adiabatic quantum transitions. Proc. R. Soc. Lond. A 429, 61-72 (1990)

9. Berry, M.V., Robbins, J.M.: Chaotic classical and half-classical adiabatic reactions: geometric magnetism and deterministic friction. Proc. R. Soc. Lond. A 442, 659-672 (1993)

10. Bornemann, F.: Homogeneization in Time of Singularly Perturbed Mechanical Systems. Lecture Notes in Math. 1687. Berlin-Heidelberg: Springer, 1998

11. Bratteli, O., Robinson, D.W.: Operator Algebras and Quantum Statistical Mechanics 1. Berlin-Heidelberg: Springer-Verlag, 1979

12. Cycon, H.L., Froese, R.G., Kirsch, W., Simon, B.: Schrödinger operators with application to quantum mechanics and global geometry. Texts and Monographs in Physics. Berlin-Heidelberg New York: Springer-Verlag, 1987

13. Davies, E.B.: Quantum theory of open systems. London: Academic Press [Harcourt Brace Jovanovich Publishers], 1976

14. Gorini, V., Kossakowski, A., Sudarshan, E.C.G.: Completely positive dynamical semigroups of $N$-level systems. J. Math. Phys. 17(5), 821-825 (1976)

15. Hagedorn, G.A., Joye, A.: Recent results on non-adiabatic transitions in quantum mechanics. In Recent advances in differential equations and mathematical physics, Volume 412 of Contemp. Math., Providence, RI: Amer. Math. Soc., 2006, pp. 183-198

16. Hille, E., Phillips, R.S.: Functional analysis and semi-groups. Providence, R.I.: Amer. Math. Soc., 1997

17. Horowitz, J., Jarzynski, C.: Exact formula for currents in strongly pumped diffusive systems. J. Stat. Phys. 136, 917-925 (2009)

18. Joye, A.: General adiabatic evolution with a gap condition. Commun. Math. Phys. 275, 139-162 (2007)

19. Kato, T.: On the adiabatic theorem of quantum mechanics. J. Phys. Soc. Japan 5, 435-439 (1950)

20. Kato, T.: Integration of the equation of evolution in a Banach space. J. Math. Soc. Japan 5(2), 208-234 (1953)

21. Kato, T.: Perturbation Theory for Linear Operators. Classics in Mathematics. Berlin, Heidelberg: Springer, 1995

22. Krein, S.G.: Linear differential equations in Banach space. Providence, RI: Amer. Mathe. Soc., 1972

23. Lindblad, G.: On the generators of quantum dynamical semigroups. Commun. Math. Phys. 48, 119130 (1976)

24. Lumer, G., Phillips, R.S.: Dissipative operators in a Banach space. Pacific J. Math. 11, 679-698 (1961)

25. Maes, C., Netočný, K., Thomas, S.R.: General no-go condition for stochastic pumping. J. Chem. Phys. 132(23), 234116 (2010)

26. Mandal, D., Jarzynski, C.: A proof by graphical construction of the no-pumping theorem of stochastic pumps. J. Stat. Mech. Th. Exp. 2011(10), P10006 (2011)

27. Nenciu, G.: Linear adiabatic theory. exponential estimates. Commun. Math. Phys. 152, 479-496 (1993)

28. Nenciu, G., Rasche, G.: On the adiabatic theorem for nonself-adjoint Hamiltonians. J. Phys. A. 25, 57415751 (1992)

29. Parrondo, J.M.R.: Reversible ratchets as Brownian particles in an adiabatically changing periodic potential. Phys. Rev. E. 57(6), 7297-7300 (1998)

30. Paz, J.P., Zurek, W.H.: Quantum limit of decoherence: Environment induced superselection of energy eigenstates. Phys. Rev. Lett. 82, 5181 (1999)

31. Rahav, S., Horowitz, J., Jarzynski, Ch.: Directed flow in nonadiabatic stochastic pumps. Phys. Rev. Lett. 101(14), 140602 (2008)

32. Reed, M., Simon, B.: Fourier Analysis and Self-Adjointness, Volume 2 of Methods of Modern Mathematical Physics. London: Academic Press, 1975 
33. Simon, B.: Trace ideals and their applications. Mathematical Society Lecture Note Series. Cambridge: Cambridge University Press, 1979

34. Stroock, D.W.: An introduction to Markov processes, Volume 230 of Graduate Texts in Mathematics. Berlin: Springer-Verlag, 2005

35. Teufel, S.: A note on the adiabatic theorem without gap condition. Lett. Math. Phys. 58(3), 255-261 (2001)

Communicated by M. Aizenman 